\title{
Features of transmission of electromagnetic waves through composite nanoresonators including epsilon-near-zero metamaterials
}

\author{
Evgenii Starodubtsev* \\ Gomel State Technical University, October ave. 48, Gomel 246746, Belarus
}

Received: 13 December 2019 / Accepted: 13 April 2020

\begin{abstract}
Transmission of electromagnetic waves through nanometric multilayers (nanoresonators) including a main composite layer made of two alternating strips of low-absorbing dielectrics that is sandwiched between epsilon-near-zero (ENZ) or metallic spacer layers has been modeled. Analytical models are based on exact solutions of electromagnetic boundary problems. The spacers with the definite properties lead to extreme dependences of amplitude transmission coefficients on the system parameters and drastic increase in phase difference of the transmitted waves. These effects are most pronounced for subwavelength multilayer thicknesses due to multibeam interference features in the nanoresonator, and they can be amplified when the main layer and (or) the whole system thicknesses decrease. The investigated transmission features take place under variations of the system parameters such as anisotropy of the main layer materials, non-ideal realization of ENZ materials, oblique incidence of the exciting radiation (for small incidence angles). The obtained results can have applications in development of ultra-thin nanophotonics devices using phase transformation of transmitted waves.
\end{abstract}

Keywords: Epsilon-near-zero metamaterial / nanoresonator / transmission / phase difference / multibeam interference

\section{Introduction}

The progress in theory and technologies of nanophotonics, plasmonics, optics of metamaterials (MMs) and metasurfaces provided possibilities to reduce dimensions of many optical elements such as modulators, filters, absorbers, phase shifters, holograms, nanoresonators and others down to nanometer values (see, e.g. recent reviews [1-12] and numerous references in these works). The main purpose of such ultra-small devices is to control the radiation characteristics (amplitude, phase, polarization) at deeply subwavelength dimensions of a composite material or system. In particular, large phase differences of two or more waves at the optical system output are required for many applications. Traditionally, the approaches based on increasing optical path difference of the waves (usage of proper waves in anisotropic media, propagation through different isotropic materials, application of optically dense materials, etc.) are used in this case. However, these methods require, as a rule, increasing geometric dimensions of optical elements (to several

\footnotetext{
* e-mail: starodub@tut.by
}

wavelengths and larger), and application of high optical density materials can lead to an undesirable high reflection or small transmission due to the large mismatch of electromagnetic properties of the device components and ambient media.

In the recent papers $[13,14]$, new possibilities to develop ultra-thin transformers of reflected waves phase differences and binary holograms on the basis of perforated multilayers including epsilon-near-zero (ENZ) MMs were revealed and investigated analytically. ENZ MMs are characterized by small values of the real and imaginary parts of permittivity, and these materials are promising for many optics applications [15-21]. In particular, ENZ components allowed implementation of new operation regimes of nanoresonators: all-optical dynamic control of transmittance [22], hybridization of ENZ modes in double nanocavities [23], engineering of ENZ resonances in metalinsulator-metal nanocavities [24]. According to the results $[13,14]$, the usage of ENZ substrates for such multilayers can lead to the extreme values of amplitude reflection coefficients depending on the system parameters. The ENZ (or metallic for thicker systems) substrate has a significant impact on the transformation of phase difference of the reflected waves. According to the modeling results, the 
optimization of this system properties allows decreasing the holograms thicknesses down to the values of the order of $10-20 \mathrm{~nm}$ as well as obtaining nanometric thickness phase transformers of reflected waves. The analysis $[13,14]$ pointed to the link between the features of interaction of electromagnetic waves with ENZ layers (for the reflection problems) and realization of regimes of effective phase transformation of the radiation using such low-dimensional systems. These results make it possible to consider the more general problem of application of ENZ MM multilayer components for phase transformation of not only reflected but also transmitted radiation.

The present paper is devoted to analytical modeling (using exact solutions of the corresponding boundary problems) of transmission of electromagnetic waves through composite multilayers of subwavelength thicknesses that include a main dielectric layer sandwiched between two ENZ MM (or metallic) spacer layers. The numerical and graphical analysis of such a nanoresonator model has the following main goals: (i) to specify the features of operation of the multilayers including ENZ MM spacers that determine new possibilities of obtaining ultrathin devices with the large phase difference and transmission of output waves; (ii) to optimize parameters of the system under consideration for typical materials of the layers. The obtained results confirm, in general, possibilities to apply such nanoresonators as effective ultracompact phase transformers of a new type that can be useful for nanophotonics applications.

The work has the following structure. The statement of the problem, solutions of electromagnetic boundary problems and some consequences of these solutions are given in Section 2. The numerical modeling and graphical analysis of the results (Sect. 3) explain the main features of amplification of transmitted waves phase difference using the ENZ or metallic spacers of the nanoresonators. In particular, the conditions are considered in detail when decreasing the main layer and (or) the whole system thickness down to significantly subwavelength values leads to increasing phase difference of the output waves and rather high transmission. Section 4 includes a brief discussion of the results and summarizes the paper. The additional calculations excluded from the main text are contained in Appendix.

\section{Statement of the problem and analytical model}

Let us investigate transmission of an electromagnetic plane monochromatic wave through a layered system under oblique incidence of the wave (Fig. 1). The multilayer scheme and geometry of the corresponding boundary problem are illustrated in Figure $1 \mathrm{a}$ and b. Layers of thicknesses $d_{l}$ (here and below indexes $\alpha, l=1,2,3, \beta$ correspond to the quantities characterizing the multilayer components in Fig. 1b) are, respectively, the upper, main, and bottom layers. The upper and bottom layers can play a role of spacers, protective or controlling layers, nanoresonator mirrors depending on applications of the system. (b)
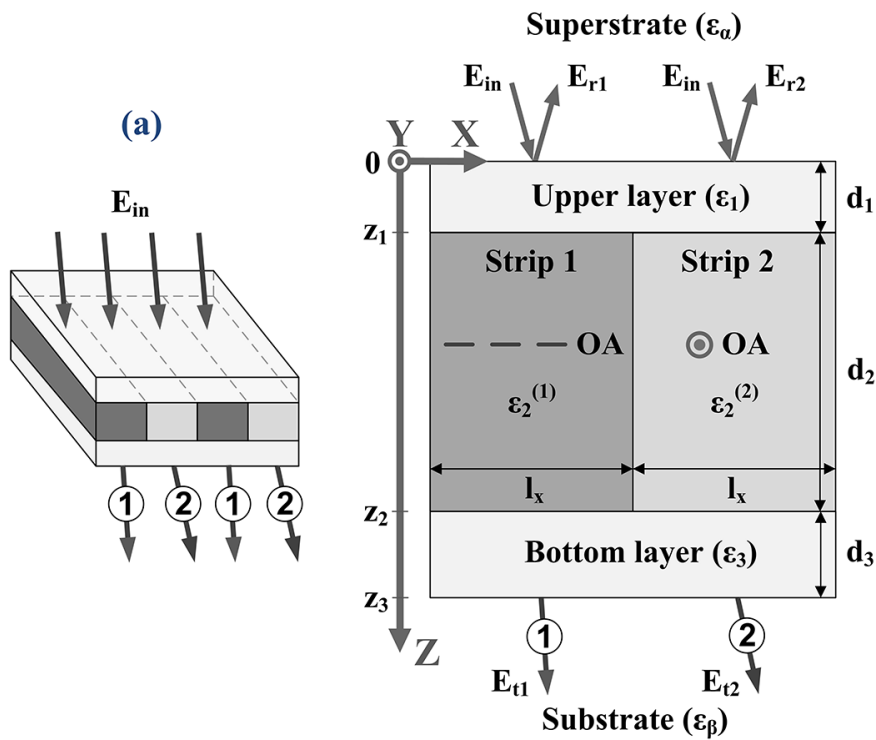

Fig. 1. (a) Scheme of the multilayer with the main composite layer formed from alternating "strips" of two different materials that can be optically uniaxial in the general case. The rays in the substrate transmitted through the multilayer are denoted by numbers 1 and $2 . \mathbf{E}_{\text {in }}$ is the incident wave field, the reflected rays in the superstrate are not shown. (b) Geometry of the corresponding electromagnetic boundary problems. The multilayer includes upper and bottom layers, which electromagnetic properties can correspond to isotropic ENZ MMs or metals, and the main layer. The superstrate and substrate materials are isotropic and optically thick. $\mathbf{E}_{\mathbf{r} 1}, \mathbf{E}_{\mathbf{r} 2}$, and $\mathbf{E}_{\mathbf{t} 1}, \mathbf{E}_{\mathbf{t} 2}$ are the fields of the waves reflected from and transmitted through Strips 1 and 2 , respectively, when the incident wave interacts with the multilayer. OA denotes the optical axis direction in the materials of Strips 1 or 2. The other denotations are given in the main text.

Media in front of the upper layer (e.g. air) and behind the bottom layer (the substrate) are assumed to be semiinfinite (optically thick). So, the reflected wave inside of the substrate is absent. Coordinate axis $\mathrm{Z}$ is perpendicular to the layers boundaries and plane XZ is the incidence plane. Phase multiplier $\exp \left[i k_{0}\left(m_{1} x+m_{3} z\right)-i \omega t\right]$ is used in expressions for the fields below where $\boldsymbol{m}=\left(m_{1}, 0, m_{3}\right)=$ $\boldsymbol{k} / k_{0}$ is a complex refraction vector [25], $\boldsymbol{k}$ is a wave vector (vectors are denoted with boldface symbols), $k_{0}=\omega / c$ is a wave number for vacuum, $i^{2}=-1, \boldsymbol{r}=(x, y, z)$ is a radiusvector. The transversal component of refraction vector is supposed to be real $\left(m_{1}\right.$ is constant at boundaries $\left.z=0, d_{l}\right)$ and the longitudinal component $m_{3}$ is complex in the general case. Electric field strengths of the incident, $\boldsymbol{E}_{i n}$, reflected, $\boldsymbol{E}_{r 1,2}$, and transmitted, $\boldsymbol{E}_{t 1,2}$, waves are considered at the boundaries $z=0$ and $z=z_{3}=d_{1}+d_{2}+d_{3}$ (Fig. 1).

Isotropic materials of the input non-absorbing medium (superstrate), absorbing layers 1, 3 (spacers), and substrate are characterized by scalar dielectric permittivities $\varepsilon_{\alpha, 1,3, \beta}$, correspondingly. The main composite layer is formed from the alternating "strips" of two different materials that are assumed to be optically uniaxial in the general case (Fig. 1). 
The Strips 1 and 2 materials are characterized by the permittivity tensors $\varepsilon_{2}^{(1)}=\operatorname{diag}\left(\varepsilon_{e}^{(1)}, \varepsilon_{o}^{(1)}, \varepsilon_{o}^{(1)}\right) \quad$ and $\varepsilon_{2}^{(2)}=\operatorname{diag}\left(\varepsilon_{o}^{(2)}, \varepsilon_{e}^{(2)}, \varepsilon_{o}^{(2)}\right)$, where $\varepsilon_{o}^{(1,2)}$ is double singular and $\varepsilon_{e}^{(1,2)}$ is non-singular complex eigenvalues of tensor $\varepsilon_{2}^{(1,2)}$ (here and below the upper indexes $(1,2)$ point to the quantities corresponding to Strips 1,2). So, the optical axis (OA, Fig. 1b) for the Strip 1 (2) material is parallel to axis $\mathrm{X}(\mathrm{Y})$, respectively.

All the multilayer components are also assumed to be absorbing (besides the superstrate) and nonmagnetic. Permittivities $\varepsilon_{\alpha, 1,3, \beta}$ or components of tensors $\varepsilon_{2}^{(1,2)}$ can have the negative real parts, all the imaginary parts of these quantities are positive according to the causality condition. So, we consider absorbing linear materials of the layers and substrate including the possible case of the MM (or metallic) layers. The denotations $a^{\prime}=\operatorname{Re}(a), a^{\prime \prime}=\operatorname{Im}(a)$ will be also used for the real and imaginary parts of scalar or vector quantities.

Let us also assume that the subwavelength thickness of the strips $d_{2}$ (along axis $\mathrm{Z}$ ) is much less than their linear sizes in plane XY: $d_{2} \ll l_{x} \ll l_{y}$ where $l_{x, y}$ are characteristic dimensions of the strips along axes $\mathrm{X}, \mathrm{Y}$ (the value of $d_{2}$ is significantly increased in Fig. 1b for illustrative purposes). The strips can be obtained with various methods and materials, e.g. they can be formed from liquid crystals with the mutually perpendicular director orientations. The similar schema of application of liquid crystals cells are used in components of display technologies for phase modulation of the transmitted radiation [26-28].

One can note that the considered geometry of inclusions (the strips) of two materials in the main composite layer is not critical for the further analysis. For example, this layer can be originated by the strips with different (unequal) values of parameters $l_{x, y}$ or by the system of parallel to axis $\mathrm{Z}$ microholes in a homogeneous host material [13,14,29]. It is important that there are, mainly, two transmitted waves at the output of the system (in the substrate), and these waves correspond to interaction of the electromagnetic field with two different materials (Strips 1,2) in the main layer.

We use the constitutive relations $\boldsymbol{D}=\varepsilon \boldsymbol{E}, \boldsymbol{B}=\mu \boldsymbol{H}$ $(\mu=1)$ and Maxwell's equations for monochromatic waves $[25]$

$$
\boldsymbol{E}=-\varepsilon^{-1}[\boldsymbol{m} \times \boldsymbol{H}], \boldsymbol{H}=[\boldsymbol{m} \times \boldsymbol{E}],
$$

where $\varepsilon^{-1}$ is the inverse to $\varepsilon$ tensor, and the dispersion equation, $\boldsymbol{m}^{\mathbf{2}}=\varepsilon$, for isotropic materials of layers 1,3 and media outside of the multilayer. Then the electric field strength of the waves in media $v=\alpha, 1,3, \beta$ (Fig. 1b) can be written as

$$
\boldsymbol{E}_{v}^{ \pm}(z)=\left(a_{v}^{ \pm}, b_{v}^{ \pm}, \mp \frac{m_{1}}{\left(m_{3}\right)_{v}} a_{v}^{ \pm}\right) \exp \left[ \pm i k_{0}\left(m_{3}\right)_{v} z\right]
$$

where (and below) upper indexes "+" and "-" correspond to the quantities characterizing transmitted and reflected proper waves in an isotropic medium (that decay in the directions along and oppositely to axis Z, respectively), and values of $\left(m_{3}\right)_{v}$ are chosen from the corresponding dispersion equations for the transmitted waves, according to the condition $\left(m_{3}^{\prime \prime}\right)_{v}>0$. In equation (2), the common for all the waves factor $\exp \left(i k_{0} m_{1} x-i \omega t\right)$ is omitted and complex scalar coefficients $a_{v}^{ \pm}, b_{v}^{\mp}$ are determined from the solutions of the boundary problems (for the substrate $a_{\beta}^{-}=b_{\beta}^{-}=0$ ). The magnetic fields strengths corresponding to equation (2) are determined by the second relation of equation (1). Coefficients $a_{v}^{ \pm}, b_{v}^{\mp}$ in equation (2) are different for the waves corresponding to interaction of the incident wave with the Strips 1 and 2 materials (e.g. for fields $\boldsymbol{E}_{\mathrm{r} 1}$ and $\boldsymbol{E}_{\mathrm{r} 2}, \boldsymbol{E}_{\mathrm{t} 1}$ and $\boldsymbol{E}_{\mathrm{t} 2}$, Fig. 1b).

The dispersion equations for uniaxial materials of the strips and corresponding TM and TE proper waves in these materials take the form

$$
\frac{m_{1}^{2}}{\varepsilon_{o}^{(1)}}+\frac{m_{3}^{2}}{\varepsilon_{e}^{(1)}}=1, \boldsymbol{E}_{T M}^{(1)}=P\left(\frac{m_{3}}{\varepsilon_{e}^{(1)}}, 0,-\frac{m_{1}}{\varepsilon_{o}^{(1)}}\right), \boldsymbol{H}_{T M}^{(1)}=P(0,1,0),
$$

$$
m_{1}^{2}+m_{3}^{2}=\varepsilon_{o}^{(1)}, \boldsymbol{E}_{T E}^{(1)}=Q(0,1,0), \boldsymbol{H}_{\mathrm{TE}}^{(1)}=Q\left(-m_{3}, 0, m_{1}\right),
$$

$$
m_{1}^{2}+m_{3}^{2}=\varepsilon_{o}^{(2)}, \boldsymbol{E}_{T M}^{(2)}=T\left(\frac{m_{3}}{\varepsilon_{o}^{(2)}}, 0,-\frac{m_{1}}{\varepsilon_{o}^{(2)}}\right), \boldsymbol{H}_{\mathrm{TM}}^{(2)}=T(0,1,0),
$$

$$
m_{1}^{2}+m_{3}^{2}=\varepsilon_{e}^{(2)}, \boldsymbol{E}_{T E}^{(2)}=R(0,1,0), \boldsymbol{H}_{\mathrm{TE}}^{(2)}=R\left(-m_{3}, 0, m_{1}\right),
$$

where equations (3), (4) and (5), (6) correspond to the materials of Strips 1 and 2, the values of $m_{3}$ are determined by the corresponding dispersion equations according to the condition $m_{3}^{\prime \prime}>0\left(m_{3}^{\prime \prime}<0\right)$ for the transmitted (reflected) waves, and the phase multiplayer $\exp \left[i k_{0}(\boldsymbol{m} \boldsymbol{r})-i \omega t\right]$ is omitted for each of the proper waves. Complex scalar coefficients $P, Q, T, R$ in equations (3)-(6) for the transmitted and reflected waves are determined from the solutions of the boundary problems.

To solve the electromagnetic boundary problems, we apply the standard approach using continuity of tangential components of the resulting fields $\boldsymbol{E}, \boldsymbol{H}$ on the multilayer boundaries. Then the following expressions for the amplitude transmission coefficients of wave 1 (transmitted through Strip 1, Fig. 1) for TM and TE polarizations of the incident wave, $t_{1}^{T M, T E}=\left|t_{1}^{T M, T E}\right| \exp \left(i \varphi_{1}^{T M, T E}\right)$, can be obtained 
See equation (7), (8) below

where the denotations for equations (2)-(6) are also used, $\eta_{\alpha, \beta, 1,3}=\left(m_{3}\right)_{\alpha, \beta, 1,3}, \eta^{T M, T E}=\left(m_{3}^{T M, T E}\right)^{(1)}$ (according to the dispersion equations from Eqs. (3) and (4)).

In a similar manner, we have the expressions for the amplitude transmission coefficients of wave 2 (transmitted through Strip 2, Fig. 1) for TM and TE polarizations of the incident wave, $t_{2}^{T M, T E}=\left|t_{2}^{T M, T E}\right| \exp \left(i \varphi_{2}^{T M, T E}\right)$ :

See equation (9), (10) below

where $\eta^{T M, T E}=\left(m_{3}^{T M, T E}\right)^{(2)}$ (according to the dispersion equations from Eqs. (5), (6)). In equations (7)-(10), the expressions for quantities $A_{ \pm}, B_{ \pm}{ }^{(1,2)}, C_{ \pm}{ }^{(1,2)}, D_{ \pm}, E_{ \pm}{ }^{(1,2)}$, $F_{ \pm}{ }^{(1,2)}$ are given in Appendix, values of $\eta_{\alpha, \beta, 1,3}, \eta^{T M, T E}$ are chosen for the transmitted waves $\left(\operatorname{Im}\left(\eta_{\alpha, \beta, 1,3}\right)>0\right.$, $\left.\operatorname{Im}\left(\eta^{T M, T E}\right)>0\right)$, and the parameters are also used: $a=\varepsilon_{\alpha} / \eta_{\alpha}, \quad b=\varepsilon_{1} / \eta_{1}, \quad c=\eta^{T M(1)} / \varepsilon_{e}^{(1)}, \quad d=\varepsilon_{3} / \eta_{3}, \quad f=\varepsilon_{\beta} / \eta_{\beta}$, $g=\eta^{T E(1)} / \eta_{1}, h=\eta_{\beta} / \eta_{3}, p=\eta^{T M(2)} / \varepsilon_{o}^{(2)}, q=\eta^{T E(2)} / \eta_{1}$.

Quantities $T_{1}^{T E, T M}=\left|t_{1}^{T E, T M}\right|^{2}, T_{2}^{T E, T M}=\left|t_{2}^{T E, T M}\right|^{2}$ can be considered as the energy transmission coefficients at the boundary $z=z_{3}$, and $\varphi_{1}^{T E, T M}, \varphi_{2}^{T E, T M}$ are the phase shifts arising in transmission of the waves corresponding to rays 1 and 2 (Fig. 1). So, the exact solutions of the boundary problems are determined by equations (7)-(10). Let us note that quantities $t_{1,2}^{T M, T E}$ in equations (7)-(10) characterize relations of the corresponding components of field $\boldsymbol{E}$.
The phase shift difference (PSD) arising for transmitted (TM or TE) waves 1 and 2 at the boundary $z=z_{3}$ and determining phase transformation effectiveness takes the form

$$
\Delta \varphi^{T M, T E}=\varphi_{1}^{T M, T E}-\varphi_{2}^{T M, T E} .
$$

Besides the exact PSD (Eq. (11)) at the multilayer output, we will also use the phase differences arising between TM or TE waves (that is, for the TM or TE exciting wave) passing one time (for one pass): (i) only through the different strips of the main layer

$$
\Delta \Phi^{T E, T M}=\left[\operatorname{Re}\left(\eta^{T M, T E(1)}\right)-\operatorname{Re}\left(\eta^{T M, T E(2)}\right)\right] k_{0} d_{2},
$$

(ii) only through the different strips of the main layer, but for the case when the thickness of these strips $\left(d_{2}\right)$ is equal to the whole multilayer thickness $\left(d_{1}+d_{2}+d_{3}\right)$, that is, when replacing layers $1,2,3$ by only the Strips 1,2 materials

$$
\begin{aligned}
& \Delta \Phi^{\sim T E, T M} \\
& \quad=\left[\operatorname{Re}\left(\eta^{T M, T E(1)}\right)-\operatorname{Re}\left(\eta^{T M, T E(2)}\right)\right] k_{0}\left(d_{1}+d_{2}+d_{3}\right) .
\end{aligned}
$$

Then relations $\Delta \varphi / \Delta \Phi, \Delta \varphi / \Delta \Phi^{\sim}$ for the corresponding polarizations characterize the relative changes of waves 1,2 PSD at the multilayer output (in presence of layers 1,2,3) in comparison with a "one pass" PSD of these waves arising

$$
t_{1}^{T M}=\frac{a_{\beta}^{+(1)} \exp \left(i k_{0} \eta_{\beta} z_{3}\right)}{a_{\alpha}^{+(1)}}=\frac{-16 a c}{\left(\frac{A_{+}}{b}+c A_{-}\right)\left(B_{+}^{(1)}-\frac{f B_{-}^{(1)}}{d}\right) \exp \left(i k_{0} \eta^{T M} d_{2}\right)+\left(c A_{-}-\frac{A_{+}}{b}\right)\left(C_{+}^{(1)}-\frac{f C_{-}^{(1)}}{d}\right) \exp \left(-i k_{0} \eta^{T M} d_{2}\right)},
$$$$
t_{1}^{T E}=\frac{b_{\beta}^{+(1)} \exp \left(i k_{0} \eta_{\beta} z_{3}\right)}{b_{\alpha}^{+(1)}}=\frac{16 \eta_{\alpha} \eta^{T E}}{\left(g D_{+}+D_{-}\right)\left(h E_{+}^{(1)}-E_{-}^{(1)}\right) \exp \left(i k_{0} \eta^{T E} d_{2}\right)+\left(g D_{+}-D_{-}\right)\left(h F_{+}^{(1)}-F_{-}^{(1)}\right) \exp \left(-i k_{0} \eta^{T E} d_{2}\right)},
$$

$$
t_{2}^{T M}=\frac{a_{\beta}^{+(2)} \exp \left(i k_{0} \eta_{\beta} z_{3}\right)}{a_{\alpha}^{+(2)}}=\frac{-16 a p}{\left(\frac{A_{+}}{b}+p A_{-}\right)\left(B_{+}^{(2)}-\frac{f B_{-}^{(2)}}{d}\right) \exp \left(i k_{0} \eta^{T M} d_{2}\right)+\left(p A_{-}-\frac{A_{+}}{b}\right)\left(C_{+}^{(2)}-\frac{f C_{-}^{(2)}}{d}\right) \exp \left(-i k_{0} \eta^{T M} d_{2}\right)},
$$

$$
t_{2}^{T E}=\frac{b_{\beta}^{+(2)} \exp \left(i k_{0} \eta_{\beta} z_{3}\right)}{b_{\alpha}^{+(2)}}=\frac{16 \eta_{\alpha} \eta^{T E}}{\left(q D_{+}+D_{-}\right)\left(h E_{+}^{(2)}-E_{-}^{(2)}\right) \exp \left(i k_{0} \eta^{T E} d_{2}\right)+\left(q D_{+}-D_{-}\right)\left(h F_{+}^{(2)}-F_{-}^{(2)}\right) \exp \left(-i k_{0} \eta^{T E} d_{2}\right)},
$$


for passing through: (i) only the main layer, equation (12); (ii) the whole multilayer filled by only materials of the strips, equation (13).

Using the obtained transmission characteristics (Eqs. (7)-(10)) and the boundary problems solutions, one can also determine quantities that serve as peculiar amplitude reflection coefficients characterizing the radiation parameters inside of the main layer relatively to ones for the incident wave. In particular, we have for the field inside of Strip 1

$$
\begin{aligned}
r_{21}^{T M} & =\frac{P^{+}}{a_{\alpha}^{+(1)}} \\
& =\frac{2 t_{1}^{T M}\left(d C_{+}^{(1)}-f C_{-}^{(1)}\right) \exp \left[i k_{0}\left(\eta_{\beta} z_{3}-\eta^{T M(1)} z_{2}\right)\right]}{B_{-}^{(1)} C_{+}^{(1)}-B_{+}^{(1)} C_{-}^{(1)}},
\end{aligned}
$$

$$
\begin{aligned}
r_{23}^{T M} & =\frac{P^{-}}{a_{\alpha}^{+(1)}} \\
& =\frac{2 t_{1}^{T M}\left(d B_{+}^{(1)}-f B_{-}^{(1)}\right) \exp \left[i k_{0}\left(\eta_{\beta} z_{3}+\eta^{T M(1)} z_{2}\right)\right]}{B_{+}^{(1)} C_{-}^{(1)}-B_{-}^{(1)} C_{+}^{(1)}},
\end{aligned}
$$

$$
\begin{aligned}
r_{21}^{T E} & =\frac{Q^{+}}{b_{\alpha}^{+(1)}} \\
& =\frac{2 t_{1}^{T E}\left(\eta_{3} F_{-}^{(1)}-\eta_{\beta} F_{+}^{(1)}\right) \exp \left[i k_{0}\left(\eta_{\beta} z_{3}-\eta^{T E(1)} z_{2}\right)\right]}{F_{-}^{(1)} E_{+}^{(1)}-F_{+}^{(1)} E_{-}^{(1)}},
\end{aligned}
$$

$$
\begin{aligned}
r_{23}^{T E} & =\frac{Q^{-}}{b_{\alpha}^{+(1)}} \\
& =\frac{2 t_{1}^{T E}\left(\eta_{3} E_{-}^{(1)}-\eta_{\beta} E_{+}^{(1)}\right) \exp \left[i k_{0}\left(\eta_{\beta} z_{3}+\eta^{T E(1)} z_{2}\right)\right]}{F_{+}^{(1)} E_{-}^{(1)}-F_{-}^{(1)} E_{+}^{(1)}},
\end{aligned}
$$

where the denotations from equations (3), (4), (7), (8) are used. Quantities $r_{21}^{T M, T E}$ and $r_{23}^{T M, T E}$ characterize reflection of the proper waves of the corresponding polarizations from the upper $\left(z=z_{1}=d_{1}\right)$ and bottom $\left(z=z_{2}=d_{1}+d_{2}\right)$ boundaries of the main layer (Fig. 1b) inside of the layer. As above, we also use energy reflection coefficients at these boundaries that are determined by the relations $R_{21}^{T E, T M}=\left|r_{21}^{T M, T E}\right|^{2}, R_{23}^{T E, T M}=\left|r_{23}^{T M, T E}\right|^{2}$. In a strict sense, quantities $r_{21,23}^{T M, T E}$ and $R_{21,23}^{T E, T M}$ are not reflection coefficients, as the numerators and denominators in equations (14)-(17) (e.g. $P^{+} / a_{\alpha}^{+(1)}$ ) are taken for the different boundaries $z=0, z_{1,2}$ without regard for the corresponding phase multipliers. However, these quantities can be used to characterize qualitatively energy distribution of the proper waves in the main layer near boundaries $z=z_{1,2}$. The similar to equations (14)-(17) relations can be obtained for the field inside of the Strip 2 material.

Using equations (3), (14), (15) and the expression for the time-averaged Pointing vector, $\boldsymbol{S}=\left(\frac{c}{8 \pi}\right) \operatorname{Re}\left[\boldsymbol{E} \times \boldsymbol{H}^{*}\right]$, where $c$ is the speed of light in vacuum and asterisk (here and below) denotes complex conjugation, one can obtain the normalized value of the resulting electromagnetic field intensity inside of the main layer (for Strip 1 and TM incident wave)

\section{See equation (18) below}

where the denotations are used: $U=\left|r_{21}^{T M}\right|^{2} \exp \left[-2 k_{0} z\right.$ $\left.\operatorname{Im}\left(\eta^{T M(1)}\right)\right]+r_{21}^{T M}\left(r_{23}^{T M}\right)^{*} \exp \left[2 i k_{0} z \operatorname{Re}\left(\eta^{T M(1)}\right)\right], V=\left|r_{23}^{T M}\right|^{2}$ $\exp \left[2 k_{0} z \operatorname{Im}\left(\eta^{T M(1)}\right)\right]+r_{23}^{T M}\left(r_{21}^{T M}\right)^{*} \exp \left[-2 i k_{0} z \operatorname{Re}\left(\eta^{T M(1)}\right)\right]$, $\boldsymbol{S}_{i n}(z=0)$ is the time-averaged Pointing vector and $\psi$ is incidence angle of the incident wave on the boundary $z=0$. Equation (18) takes into account multibeam interference of the opposite transmitted and reflected TM proper waves inside of the main layer.

The standard relations for phase incursion of the waves inside of the resonator are used usually to determine conditions when transmission maxima of the similar nanoresonators are realized (e.g. $[22,30])$. However, obtaining compact analytical expressions for phases of the transmitted waves is not trivial in the case under consideration. So, the detailed numerical analysis of phases, amplitude and energy transmission coefficients of the output waves will be executed below using equations (7)-(13).

Obviously, the obtained simplified model considers only two "ways" of the transmission in the system that correspond to "conventional" transmission of rays 1 and 2 (Fig. 1). Clearly, a part of the radiation also reflects from the strips vertical walls and propagates from one to other strip with the incidence angle $\psi$ growth. Moreover, the analytical model does not account for the strips form, their location in plane XY, and possible excitation of additional near-field modes including the effects of extraordinary interaction of radiation with the system (under conditions of surface plasmons excitation for the ordered inclusions arrays) [31-34]. However (similarly to the reflection models $[13,14]$ ), the multilayer (Fig. 1) includes dielectric strips with the deeply subwavelength thickness $\left(d_{2} \ll \lambda\right)$ and "macroscopic" dimensions in plane XY $\left(\lambda<l_{x} \ll l_{y}\right)$. For optical applications, the values of $l_{x, y}$ (or other parameters characterizing sizes of the main layer inhomogeneities and distances between them in plane $\mathrm{XY}$ ) can be estimated as several microns or larger. Exactly that and the condition of small incidence angles allow the use of transmission coefficients (Eqs. (7)-(10)) for the main part of the transmitted radiation, and without application of more complicated diffraction theory methods (e.g. [35-38]). The similar approach (based on exact analytical solutions of the boundary problems) was

$$
I^{T M(1)}=\frac{\left.\mid \boldsymbol{S}\left(z_{1} \leq z \leq z_{2}\right)^{(1)}\right] \mid}{\left|S_{\text {in }}(z=0)\right|}=\frac{\cos ^{2} \psi}{\sqrt{\varepsilon_{\alpha}}} \sqrt{\left\{\operatorname{Re}\left[\frac{m_{1}}{\varepsilon_{o}^{(1)}}(U+V)\right]\right\}^{2}+\left\{\operatorname{Re}\left[\frac{\eta^{T M(1)}}{\varepsilon_{e}^{(1)}}(U-V)\right]\right\}^{2}}
$$


recently confirmed experimentally for calculation of the waves reflected from the nanometric binary holograms [29].

\section{Numerical and graphical analysis}

\subsection{Main assumptions and parameters}

The main goal of the analysis below is to determine parameters of the investigated systems when rather large values of PSD and transmission of waves 1, 2 take place for the minimal main layer or whole multilayer thicknesses. According to equations (11)-(13), it means that we consider the cases when $\Delta \varphi / \Delta \Phi>1, \Delta \varphi / \Delta \Phi^{\sim}>1$, that is, when it is possible to "amplify" PSD of waves 1,2 in comparison with the "one pass" PSD corresponding to the one-time run of the radiation through the main layer or the whole system. Indexes TM, TE are further omitted when both types of the waves are considered or the choice of the waves is clear from the context.

We choose some model ENZ MMs and silver as a metallic component frequently used in photonics applications for the materials of layers 1, 3 (the upper and bottom layers, Fig. 1). The cases are considered when the main layer is formed from the strips of transparent (lowabsorbing) dielectrics (the numerical analysis shows that it is a rather optimal case). For all the figures below, air is assumed to be the superstrate and substrate medium $\left(\varepsilon_{\alpha, \beta}=1\right)$, and the spacer layers thicknesses are equal $\left(d_{1}=d_{3}\right)$. The effects under consideration also take place for the general (less "symmetric") cases when $\varepsilon_{\alpha} \neq \varepsilon_{\beta}, d_{1} \neq d_{3}$.

The main features of the nanoresonators occur for both oblique (for small incidence angles) and normal incidence of the exciting wave (when the difference between TE and TM waves disappears). Isotropic dielectrics are chosen as the main layer materials for many figures below (Figs. 2-10, Sect. 3.2). The impact of optical anisotropy of the strips materials (that is not a determining factor for the effects under investigation within the considered parameter ranges) is characterized by Figures 11, 12 data and numerical estimations given below (Sect. 3.3). For all the graphs in Figures 2-10, the parameters are chosen: $\psi=0$ (besides the Fig. 8 data), $\varepsilon_{o}^{(1)}=\varepsilon_{e}^{(1)}=2.5+10^{-3} i$, $\varepsilon_{o}^{(2)}=\varepsilon_{e}^{(2)}=1$ (the Strip 2 material is air). The other parameter values are given in the figures captions.

Phase shifts in the graphs further are calculated in relative units of radians $/ \pi$. Phases $\varphi_{1,2}$ are determined below using built-up functions of the computer algebra system (Mathcad, PTC) and values of $t_{1,2}$ (Eqs. (7)-(10)). As these built-up functions return the principal argument of a complex number (between $-\pi$ and $\pi$ ), so phase values "jumps" can be in graphs of dependences of quantities $\varphi_{1,2}$ on the system parameters when phase takes values $\pm \pi$.

\subsection{Case of isotropic main layer materials}

The data in Figure 2 illustrate phase and energy characteristics of the transmitted waves depending on the main layer thickness for the following choice of the parameters of layers 1, 3: "conventional" layers (Fig. 2a,d); one of the layers is conventional, and the other one is ENZ
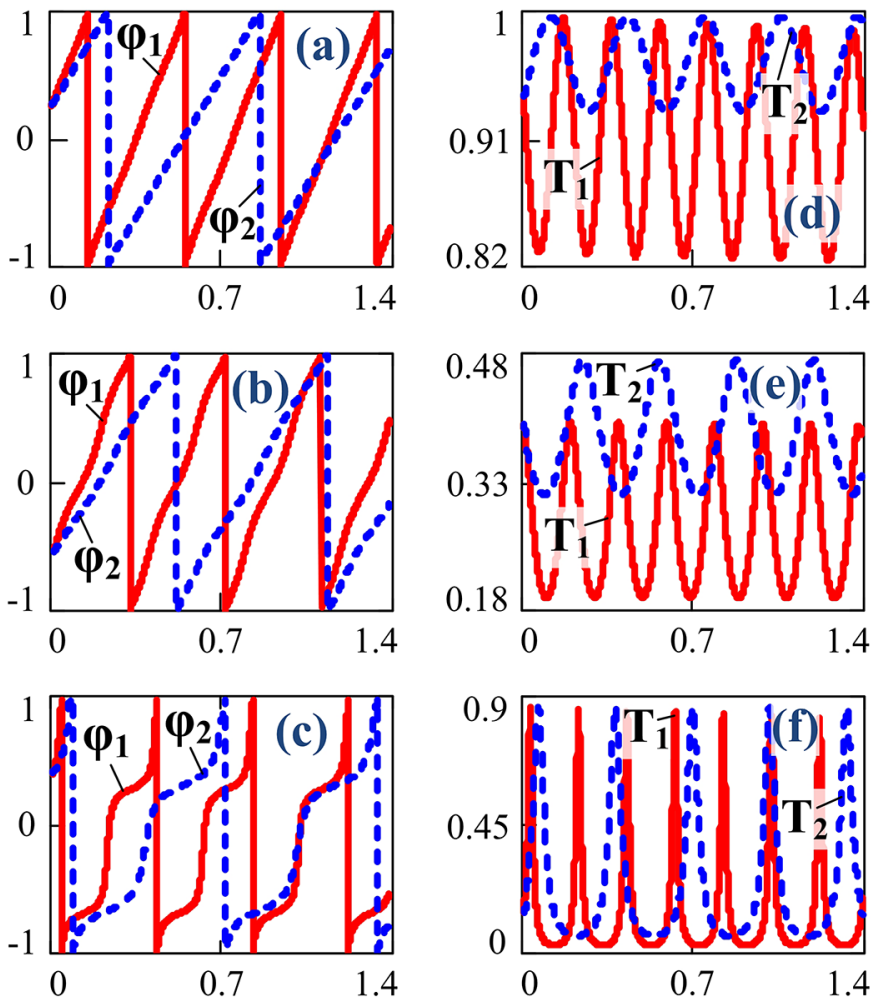

\section{$\mathbf{d}_{2}, \mu \mathbf{m}$}

Fig. 2. Dependences of phases $\varphi_{1,2}(\mathrm{a}-\mathrm{c})$ and energy transmission coefficients $T_{1,2}(\mathrm{~d}-\mathrm{f})$ of waves 1,2 on the main layer thickness $d_{2}$ for various materials of layers 1,3 . The values of parameters are: $\varepsilon_{1}=\varepsilon_{3}=2+10^{-3} i \quad(\mathrm{a}, \mathrm{d}) ; \quad \varepsilon_{1}=2+10^{-3} i, \quad \varepsilon_{3}=0.01+0.01 i \quad(\mathrm{~b}, \mathrm{e}) ;$ $\varepsilon_{1}=\varepsilon_{3}=0.01+0.01 i(\mathrm{c}, \mathrm{f})$. The values of parameters for all the graphs are: $d_{1,3}=0.25 \mu \mathrm{m}, \lambda=0.63 \mu \mathrm{m}$. Parameter $d_{2}$ is used as the abscissa axis for all the graphs.

(Fig. 2b,e); both the layers are ENZ (Fig. 2c,f). When thickness $d_{2}$ is varying, the phases and transmission coefficients modulation is significantly amplified in the transition from "conventional" to ENZ layers 1, 3. Figure 2b, e data are "intermediate" in comparison with the cases of "conventional" and ENZ layers 1, 3, and the corresponding graphs do not change when the layers properties are rearranged: $\varepsilon_{1} \leftrightarrow \varepsilon_{3}$. When the values of $d_{2}$ change, for the case of ENZ layers 1, 3, the additional modulation of quantities $\varphi_{1,2}$ and periodic resonance changes of quantities $T_{1,2}$ from 0 up to 0.9 are realized (the data in Fig. 2c,f in comparison with ones in Fig. 2a,d). It is seen that positions of characteristic points in the graphs (phase jumps, transmission extrema) depend strongly on the layers 1, 3 material. The distances between the neighboring abrupt changes of $\varphi_{1,2}$ values from $\pi$ to $-\pi$ (Fig. 2a-c) and maxima of functions $T_{1,2}\left(d_{2}\right)$ (Fig. 2d-f) are well described by constant values $\Delta d_{2}$ and $\Delta d_{2} / 2$, correspondingly, where $\Delta d_{2}=\lambda / \sqrt{\operatorname{Re}\left(\varepsilon_{2}^{(1,2)}\right)}$. So, these properties for each of the nanoresonators corresponding to Strips 1 and 2 (for low-absorbing main layer materials) are 
similar to ones for the "ideal" Fabry-Perot resonator [39] and have the pronounced interference nature. One can see that the presence of both ENZ layers 1, 3 leads to the significant amplification of the interference effects.

Changes of quantities $t_{1,2}^{\prime \prime \prime}$ in the transition from "conventional" to ENZ layers 1, 3 (for the data of Fig. 2a,d and c,f) are illustrated in Figure 3. For "conventional" layers 1,3 , the graphs of functions $t_{1,2}^{\prime \prime \prime}\left(d_{2}\right)$ are rather smooth and periodic (Fig. 3a). It is seen, that the resonance character of the dependences in Figure $2 \mathrm{c}, \mathrm{f}$ is due to the similar features of graphs $t_{1,2}^{\prime \prime \prime}\left(d_{2}\right)$ (Fig. $3 \mathrm{~b}$ ). For the case of ENZ layers 1, 3, the trajectories of the tips of vectors $\boldsymbol{t}_{1,2}=\left(t_{1,2}^{\prime}, t_{1,2}^{\prime \prime}\right)$ on the complex plane change strongly and similarly when thickness $d_{2}$ is varied (Fig. 3c-f). For quantities $\boldsymbol{t}_{1,2}$ characterizing waves 1,2 , the trajectory form changes cardinally (Fig. 3c,e in comparison with Fig. 3d,f). The data in Figure $3 \mathrm{~g}, \mathrm{~h}$ illustrate the trajectories of vectors $\boldsymbol{t}_{1,2}$ tips for changes of parameters $\varepsilon_{1}^{\prime}=\varepsilon_{3}^{\prime}$ in the range $(-2 ; 2)$ when $d_{2}=39 \mathrm{~nm}$. For the values of the order $\varepsilon_{1,3}^{\prime}=-0.2 \div 0.2$ the tip of vector $\boldsymbol{t}_{1}$ "moves" on the complex plane much "faster" than one for vector $\boldsymbol{t}_{2}$ providing larger values of $|\Delta \varphi|$ for ENZ layers 1,3 . However, the trajectories of the tips of vectors $\boldsymbol{t}_{1,2}$ for changing $\varepsilon_{1,3}^{\prime}$ are similar (Fig. 3g,h). In Figure $3 \mathrm{c}-\mathrm{h}$ graphs, there are denotations of the characteristic points (values of $d_{2}$, Fig. 3c-f, and parameters $\varepsilon_{1,3}^{\prime}$, Fig. $3 \mathrm{~g}, \mathrm{~h}$ ) describing the "motion" of vectors $\boldsymbol{t}_{1,2}$ tips for increasing $d_{2}$ or $\varepsilon_{1,3}^{\prime}$.

Figure 4 illustrates phase differences $\left(\Delta \varphi, \Delta \Phi, \Delta \Phi^{\sim}\right)$ and transmission coefficients $\left(T_{1,2}\right)$ of waves 1,2 for "conventional" and ENZ layers 1, 3 depending on the system parameters. For "conventional" layers 1, 3, quantities $T_{1,2}$ depend weakly on parameters $d_{2}$ and $\lambda$ (when neglecting frequency dispersion of the multilayer components), and quantities $\Delta \varphi, \Delta \Phi$ have close values (Fig. 4a,b). So, in this case PSD of waves 1 and 2 at the boundary $z=z_{3}$ coincides practically with "one pass" phase difference $\Delta \Phi$ (Eq. (12)). The situation changes drastically when choosing ENZ layers 1, 3 for rather thin (subwavelength) main layers (Fig. $4 \mathrm{c}-\mathrm{h}$ ). With that, the ranges of parameters $d_{2}, \lambda, d_{1,3}, \varepsilon_{1,3}^{\prime}$ values can be realized when the condition $\Delta \varphi \gg \Delta \Phi$ is satisfied. So, ENZ layers 1, 3 (isotropic and the same ones for Strips 1,2) lead to the drastic increase of waves 1, 2 PSD at the multilayer output in comparison with quantity $\Delta \Phi$. In this case, the strong dependences of quantities $T_{1,2}$ on the system parameters also take place (Fig. 4c,d,f,g). It is important that large values of $\Delta \varphi$ and $\Delta \varphi / \Delta \Phi$ can be obtained for the rather high transmission. For example, the first crossing of the functions $T_{1,2}\left(d_{2}\right)$ graphs in Figure $4 \mathrm{c}$ (for increasing $d_{2}$ ) corresponds to the main layer thickness $d_{2}=39 \mathrm{~nm}$ when $\Delta \varphi \approx \pi / 2, \Delta \varphi / \Delta \Phi \approx 7$ (Fig. 4e), $T_{1,2} \approx 0.43$. The corresponding point is also pointed in Figure $3 \mathrm{c}-\mathrm{f}$, and this value of $d_{2}$ is used for the data in Figure $3 \mathrm{~g}$, h. The graphs of functions $\Delta \varphi / \Delta \Phi\left(d_{2}\right), \Delta \varphi / \Delta \Phi\left(d_{1,3}\right), \Delta \varphi\left(\varepsilon_{1,3}^{\prime}\right)$ have the pronounced maxima near values $d_{2}=36 \mathrm{~nm}, d_{1,3}=330 \mathrm{~nm}$, $\varepsilon_{1,3}^{\prime} \approx 0$, respectively (Fig. $4 \mathrm{e}-\mathrm{h}$ ). Let us note that condition $\Delta \varphi / \Delta \Phi^{\sim}<1$ takes place for all the data in Figure 4 (that characterize the case of a rather large dielectric contrast
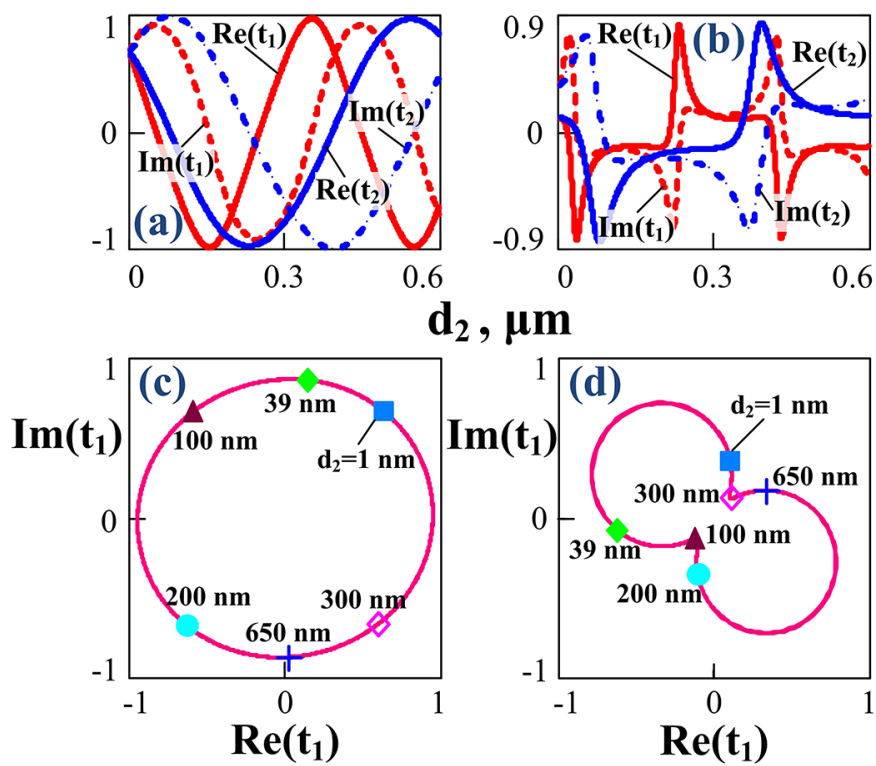

1 (d)
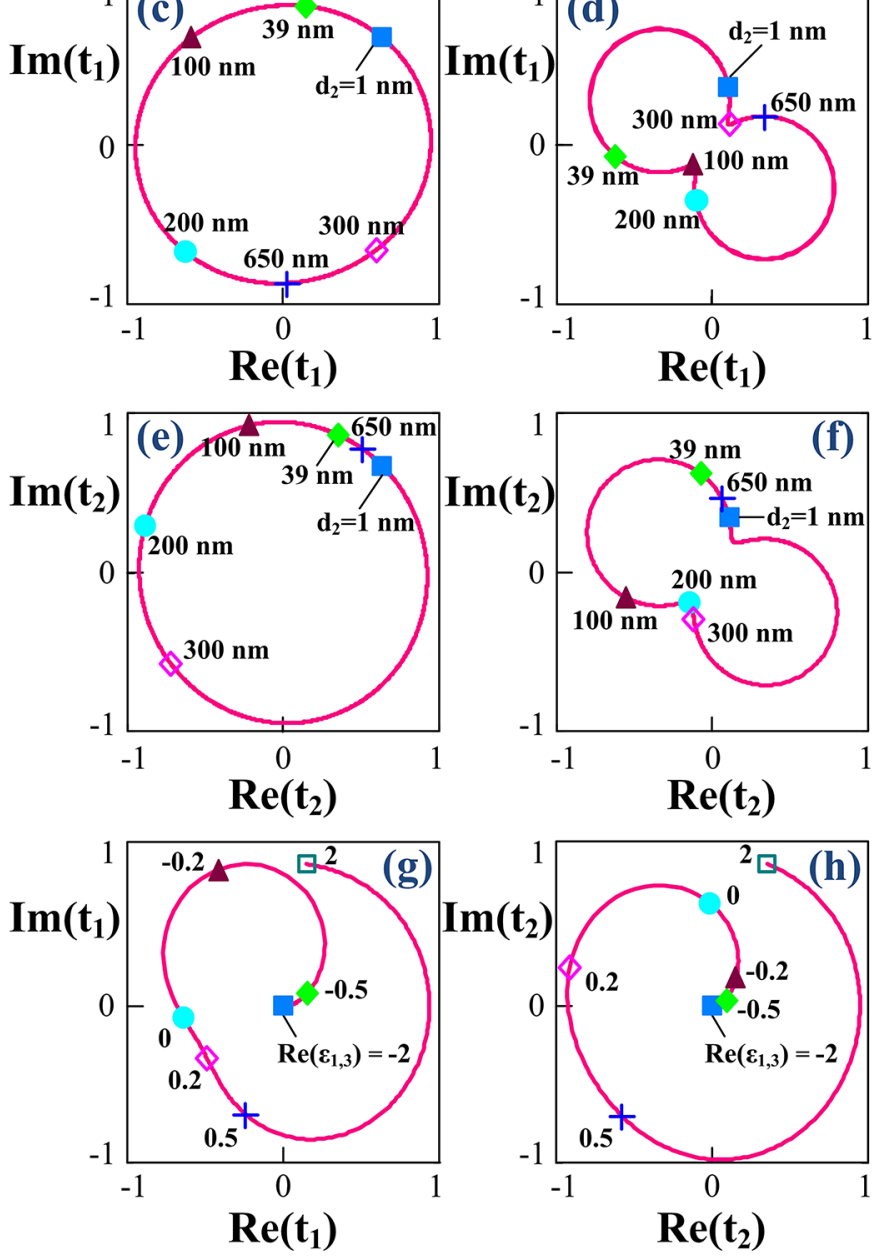

Fig. 3. The effect of parameter $d_{2}$ on the real and imaginary parts of amplitude transmission coefficients of waves $1,2(\mathrm{a}, \mathrm{b})$ and trajectories of the tips of vectors $t_{1,2}$ on the complex plane depending on parameters $d_{2}(\mathrm{c}-\mathrm{f})$ and $\varepsilon_{1,3}^{\prime}(\mathrm{g}, \mathrm{h})$. The values of parameters are: $\varepsilon_{1}=\varepsilon_{3}=2+10^{-3} i(\mathrm{a}, \mathrm{c}, \mathrm{e}) ; \varepsilon_{1}=\varepsilon_{3}=0.01+0.01 i$ (b, d, f); $\varepsilon_{1,3}^{\prime \prime}=10^{-3}, d_{2}=39 \mathrm{~nm}(\mathrm{~g}, \mathrm{~h})$. The values of parameters for all the graphs are: $d_{1,3}=0.25 \mu \mathrm{m}, \lambda=0.63 \mu \mathrm{m}$.

between isotropic materials of the strips: $\left|\varepsilon_{2}^{(1)}-\varepsilon_{2}^{(2)}\right| \approx 1.5$ ). So, the fulfilment of conditions $\Delta \Phi \ll \Delta \varphi<\Delta \Phi^{\sim}$ is possible for the considered parameters (for rather small values of $d_{2}$ and optimally selected values of $d_{1,3}$ for ENZ layers 1,3$)$. For these data and small dielectric contrasts $\left(\left|\varepsilon_{2}^{(1)}-\varepsilon_{2}^{(2)}\right|<0.5\right)$, the numerical analysis also showed the simultaneous fulfilment of more optimal conditions of PSD amplification: $\Delta \varphi / \Delta \Phi>15,1<\Delta \varphi / \Delta \Phi^{\sim}<2$. 

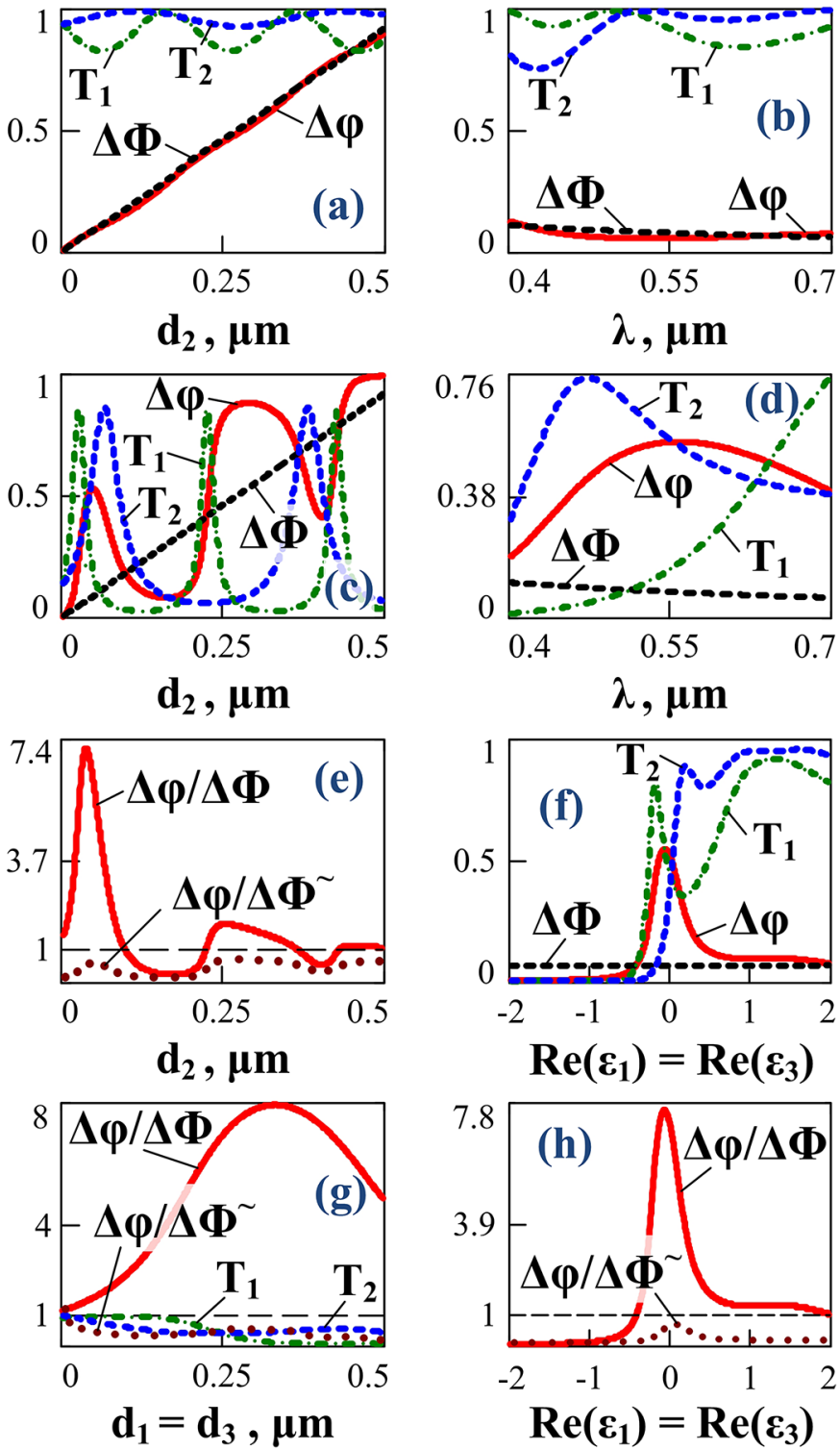

Fig. 4. Dependences of PSD $\Delta \varphi, \Delta \Phi$, relations $\Delta \varphi / \Delta \Phi, \Delta \varphi / \Delta \Phi^{\sim}$ and energy transmission coefficients $T_{1,2}$ of waves 1,2 on the main layer thickness $d_{2}$ (a, c, e), wavelength $\lambda$ (b, d), parameters $\varepsilon_{1,3}^{\prime}(\mathrm{f}, \mathrm{h}), d_{1,3}(\mathrm{~g})$ for various materials of layers 1,3 . The values of parameters are: $\varepsilon_{1}=\varepsilon_{3}=2+10^{-3} i(\mathrm{a}, \mathrm{b}) ; \varepsilon_{1}=\varepsilon_{3}=0.01+0.01 i$ $(\mathrm{c}, \mathrm{d}, \mathrm{e}, \mathrm{g}) ; d_{2}=39 \mathrm{~nm}(\mathrm{~b}, \mathrm{~d}, \mathrm{f}, \mathrm{h}) ; \varepsilon_{1,3}^{\prime \prime}=0.01$ (f, h). The values of parameters for all the graphs are: $d_{1,3}=0.25 \mu \mathrm{m}$ (besides g); $\lambda=0.63 \mu \mathrm{m}$ (besides $\mathrm{b}, \mathrm{d}$ ). Figures e and $\mathrm{h}$ detail the data in Figures $\mathrm{c}$ and $\mathrm{f}$, respectively. The black horizontal dashed line in Figures e, g, h characterize equality to 1 of the corresponding quantities.

The considered effects are also realized for larger values of parameter $\operatorname{Re}\left(\varepsilon_{2}^{(1)}\right)$ (e.g. for $\left.\operatorname{Re}\left(\varepsilon_{2}^{(1)}\right) \sim 10\right)$. However, in this case the satisfaction of condition $\Delta \varphi \gg \Delta \Phi$ worsens (as the straight line $\Delta \Phi\left(d_{2}\right)$ slope increases), and it is difficult to obtain simultaneously large values $T_{1,2}$ (as the maxima of functions $T_{1}\left(d_{2}\right)$ and $T_{2}\left(d_{2}\right)$ move apart with the growth of parameter $\left.\left|\varepsilon_{2}^{(1)}-\varepsilon_{2}^{(2)}\right|\right)$. Increasing parameter $\operatorname{Im}\left(\varepsilon_{2}^{(1)}\right)$ is
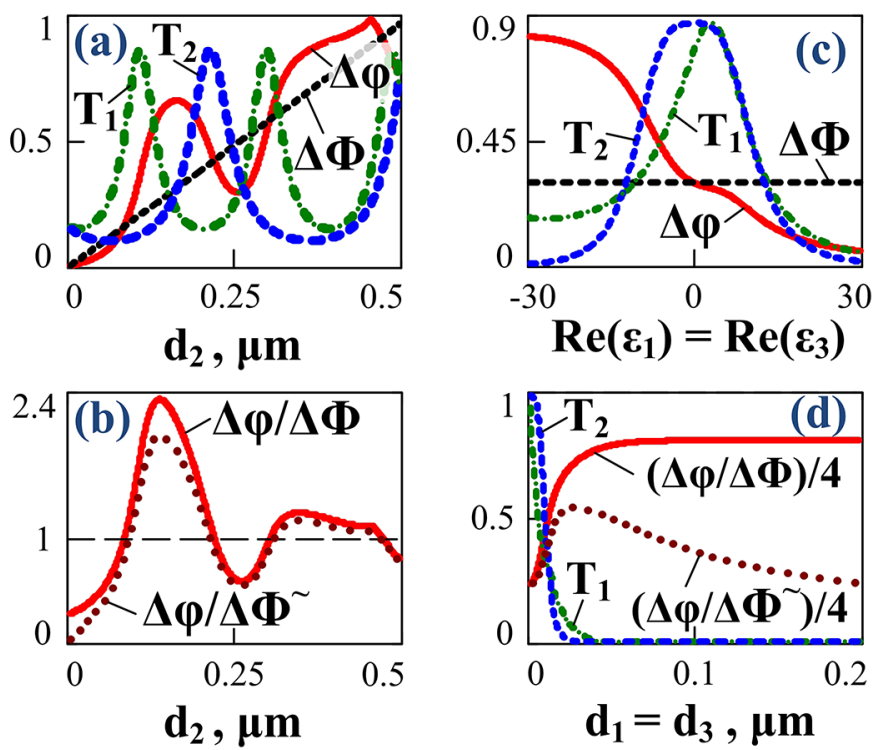

Fig. 5. Dependences of $\operatorname{PSD} \Delta \varphi, \Delta \Phi$, relations $\Delta \varphi / \Delta \Phi, \Delta \varphi / \Delta \Phi^{\sim}$ and energy transmission coefficients $T_{1,2}$ of waves 1,2 on the main layer thickness $d_{2}(\mathrm{a}, \mathrm{b})$, parameters $\varepsilon_{1,3}^{\prime}(\mathrm{c}), d_{1,3}(\mathrm{~d})$ for a metallic material of layers 1,3 . The values of parameters are: $\varepsilon_{1}=\varepsilon_{3}=$ $-13+0.5 i(\mathrm{a}, \mathrm{b}, \mathrm{d}) ; d_{2}=156 \mathrm{~nm}(\mathrm{c}, \mathrm{d}) ; \varepsilon_{13}^{\prime \prime}=0.5(\mathrm{c})$. The values of parameters for all the graphs are: $d_{1,3}=13 \mathrm{~nm}$ (besides $\mathrm{d}$ ), $\lambda=0.6 \mu \mathrm{m}$. Figure b details the data in Figure a. The black horizontal dashed line in fig. b characterizes equality to 1 of the corresponding quantities.

also not optimal, as it leads to decreasing waves 1,2 transmission.

The graphs in Figure 5 characterize the multilayer corresponding to Figure 4 data but for "metallic" layers 1, 3 and rather optimal (for realization of large PSD and transmission of waves 1,2 ) parameters $d_{1,2,3}$. Qualitatively, the graphs in Figure 5a,b are similar to ones in Figure 4c,e. In particular, with the growth of values $d_{2}$, the first crossing of the functions $T_{1,2}\left(d_{2}\right)$ graphs in Figure 5 a realizes for $d_{2}=156 \mathrm{~nm}$, when $\Delta \varphi \approx 0.7 \pi, \Delta \varphi / \Delta \Phi \approx 2, \Delta \varphi / \Delta \Phi^{\sim} \approx 2$ (Fig. $5 \mathrm{~b}$ ), $T_{1,2} \approx 0.26$. However, functions $\Delta \varphi\left(\varepsilon_{1,3}^{\prime}\right)$ $\left(\Delta \varphi / \Delta \Phi\left(d_{1,3}\right)\right)$ have no maxima and decrease (increase) monotonically for the considered parameters values (Fig. 5c,d). That explains the choice of rather small (negative) values of $\varepsilon_{1,3}^{\prime \prime}$ and thicknesses $d_{1,3}$ for realization of large values of $\Delta \varphi, T_{1,2}$. With that, we have the condition $d_{2} \ll d_{1,3}$ for ENZ layers 1, 3 (Fig. 4 ), and the opposite one $d_{2} \gg d_{1,3}$ for "metallic" layers 1, 3 (Fig. 5).

The transmission characteristics of waves 1, 2 depending on parameter $d_{2}$ for the case of "metallic" layers 1, 3 are illustrated in Figure 6. The comparison of the graphs in Figures 2, 6 shows that functions $\varphi_{1,2}\left(d_{2}\right), T_{1,2}\left(d_{2}\right)$ have a number of similar features for one or two layers 1,3 of ENZ or "metallic-like" materials (comparing Fig. 2b, e, f and Fig. 6a, c, d). This leads to the qualitative features considered above for the data in Figure 5. As for the data in Figure 2b, the graphs in Figure 6a,c do not change for the layers properties rearrangement: $\varepsilon_{1} \leftrightarrow \varepsilon_{3}$.

The graphical analysis above illustrates two main "regimes" of operation of the multilayers depending on 

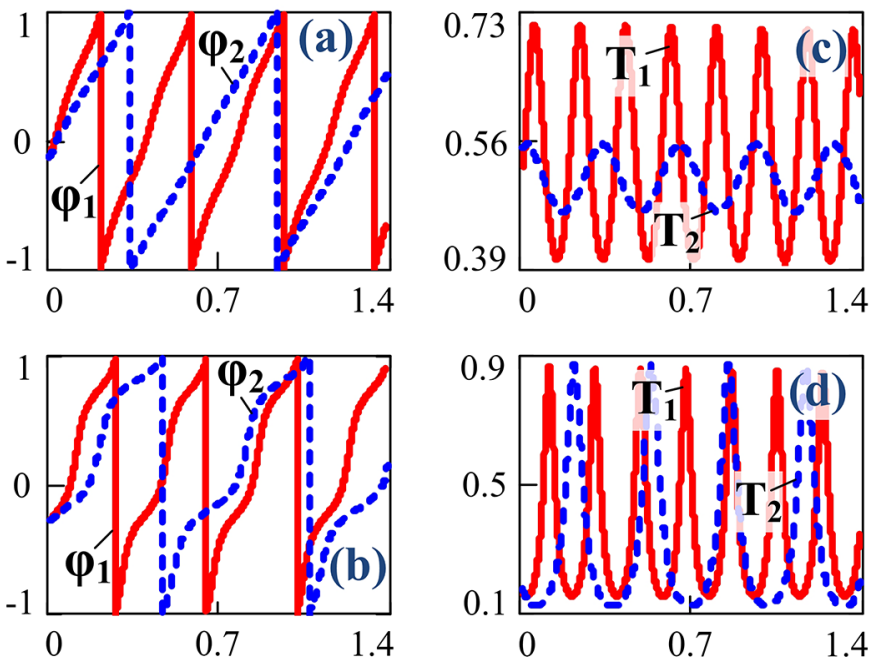

\section{$\mathbf{d}_{2}, \boldsymbol{\mu m}$}

Fig. 6. Dependences of phase differences $\varphi_{1,2}(\mathrm{a}, \mathrm{b})$ and energy transmission coefficients $T_{1,2}(\mathrm{c}, \mathrm{d})$ of waves 1,2 on the main layer thickness $d_{2}$ for various materials of layers 1,3 . The values of parameters are: $\varepsilon_{1}=2+10^{-3} i, \varepsilon_{3}=-13+0.5 i$ (a, c); $\varepsilon_{1}=\varepsilon_{3}=$ $-13+0.5 i(\mathrm{~b}, \mathrm{~d})$. The values of parameters for all the graphs are: $d_{1,3}=13 \mathrm{~nm}, \lambda=0.6 \mu \mathrm{m}$. Parameter $d_{2}$ is used as the abscissa axis for all the graphs.

layers 1 and 3 properties: for ENZ and metallic materials of the layers. These regimes can be realized for both the different and the same material of layers 1, 3 (assuming these layers to be similar). In particular, one material for two regimes can be used for the different wavelengths of the exciting radiation taking into account frequency dispersion of the dielectric properties. The data in Figure 7 illustrate possibility to use silver as the layers 1,3 material. The literary data contain a rather wide variation of the $\mathrm{Ag}$ permittivity values subject to fabrication methods, thicknesses of the layers, etc. For the numerical analysis, the following values were used: $\varepsilon_{A q}=2.27 \times 10^{-3}+0.08 i$, $-19.74+0.85 i$ for $\lambda=292,650 \mathrm{~nm}$, correspondingly [40]. The graphs in Figure 7a, b, c (the ENZ regime) and Figure $7 \mathrm{~d}, \mathrm{e}, \mathrm{f}$ (the metallic regime) well correspond to ones in Figure 4c, e, g and Figure 5a, b, d, respectively. In particular, for increasing values of $d_{2}$, the first crossing of the graphs $T_{1,2}\left(d_{2}\right)$ in Figure $7 \mathrm{a}$ and $\mathrm{d}$ corresponds to the following values of parameters: $d_{2}=19 \mathrm{~nm}, \Delta \varphi \approx 0.5 \pi$, $\Delta \varphi / \Delta \Phi \approx 6, \Delta \varphi / \Delta \Phi^{\sim} \approx 0.5, \quad T_{1,2} \approx 0.2$ and $d_{2}=171 \mathrm{~nm}$, $\Delta \varphi \approx 0.6 \pi, \Delta \varphi / \Delta \Phi \approx 2, \Delta \varphi / \Delta \Phi^{\sim} \approx 2, T_{1,2} \approx 0.3$. So, silver layers 1,3 can provide the realization of the system under investigation for two considered operation regimes.

Dependences of the main transmission characteristics of the multilayers on the incidence angle (under $\psi=0 \div \pi / 10$ and TE, TM polarizations of the incident wave) for the parameters corresponding to Figure 7 data are illustrated in Figure 8. The graphs in Figure 8a-d and e-h correspond to the ENZ and metallic regimes, respectively, for silver as the layers 1, 3 material. The thicknesses $d_{1,2,3}$ for the data in Figure 8 correspond to the first crossing of the graphs $T_{1,2}\left(d_{2}\right)$ in Figure $7 \mathrm{a}$ and $\mathrm{d}$, when rather large values of
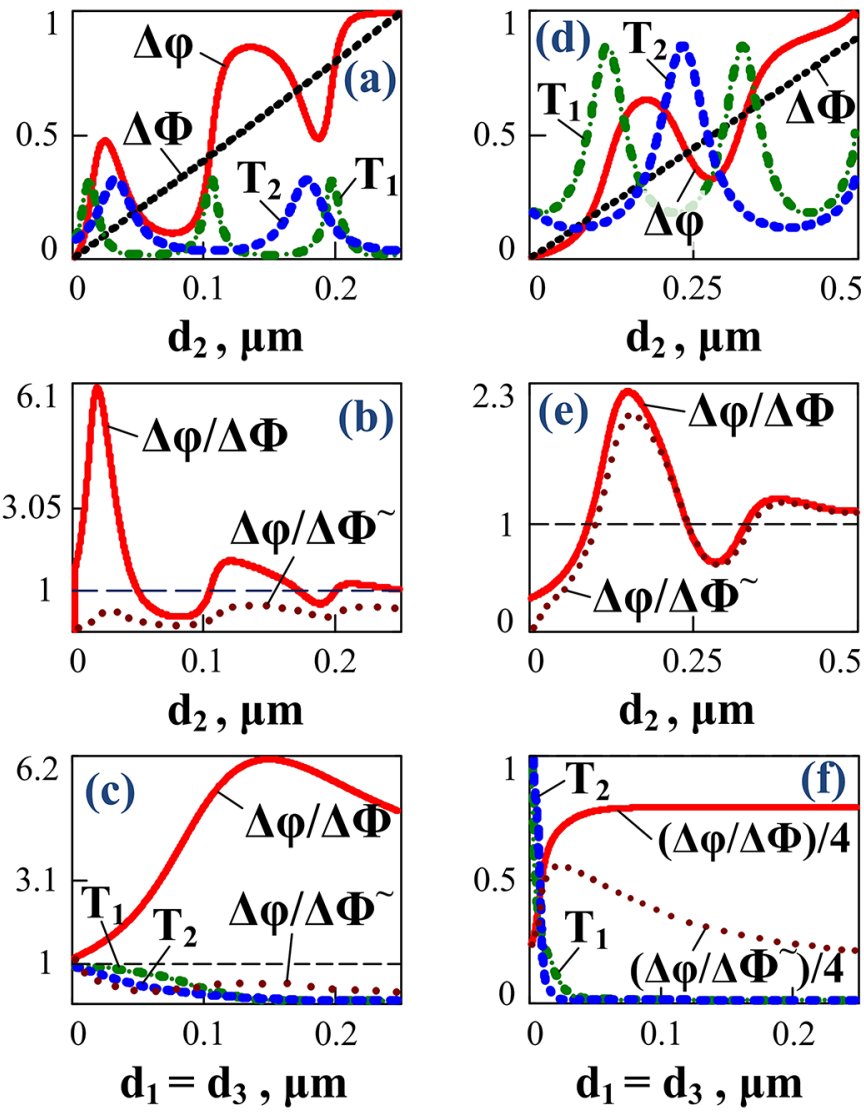

Fig. 7. Dependences of PSD $\Delta \varphi, \Delta \Phi$, relations $\Delta \varphi / \Delta \Phi, \Delta \varphi / \Delta \Phi^{\sim}$ and energy transmission coefficients $T_{1,2}$ of waves 1,2 on thicknesses $d_{2}(\mathrm{a}, \mathrm{b}, \mathrm{d}, \mathrm{e}), d_{1,3}(\mathrm{c}, \mathrm{f})$ for silver layers 1,3 and two different wavelengths of the incident wave. The values of the $\mathrm{Ag}$ permittivity for $\lambda=292 \mathrm{~nm}(\mathrm{a}, \mathrm{b}, \mathrm{c}), 650 \mathrm{~nm}(\mathrm{~d}, \mathrm{e}, \mathrm{f})$ are given in the text. The values of parameters are: $d_{1,3}=125 \mathrm{~nm}(\mathrm{a}, \mathrm{b}), 9 \mathrm{~nm}$ $(\mathrm{d}, \mathrm{e}) ; d_{2}=19 \mathrm{~nm}(\mathrm{c}), 171 \mathrm{~nm}(\mathrm{f})$. Figures $\mathrm{b}$ and e detail the data in figures a and $\mathrm{d}$, respectively. The black horizontal dashed line in figures b, c, e characterizes equality to 1 of the corresponding quantities.

parameters $\Delta \varphi, \Delta \varphi / \Delta \Phi, \Delta \varphi / \Delta \Phi^{\sim}, T_{1,2}$ take place simultaneously. With growth of the incidence angle, phase characteristics $\Delta \varphi, \Delta \varphi / \Delta \Phi$ have larger values and change (decrease) stronger in the case of ENZ layers 1, 3 and TM polarization of the incident wave (the left panels of Fig. 8 in comparison with the right ones). Parameters $\Delta \Phi$ and $\Delta \varphi / \Delta \Phi^{\sim}$ change rather weakly for all the data in Figure 8 . For angles $\psi=0 \div \pi / 20$, quantities $T_{1,2}$ have the comparable values for both ENZ (Fig. 8a,c) and metallic (Fig. 8e,g) layers 1, 3. Thus, Figure 8 data characterize the possibilities of simultaneous realization of rather large values of PSD and transmission for both the normal and oblique incidence of the incident radiation on the ultra-thin multilayers (for rather small $\psi$ values).

According to the similar analysis (the data are not shown), Figures $2-5$ data do not change qualitatively for cases of anisotropic materials of the strips (for rather small anisotropy values of the strips material, e.g. for parameters $\left.\varepsilon_{o}^{(1,2)}=2.6+10^{-3} i ; \varepsilon_{e}^{(1,2)}=2.2+2 \times 10^{-3} i\right)$. These data changes are also very small for oblique incidence and TM or 

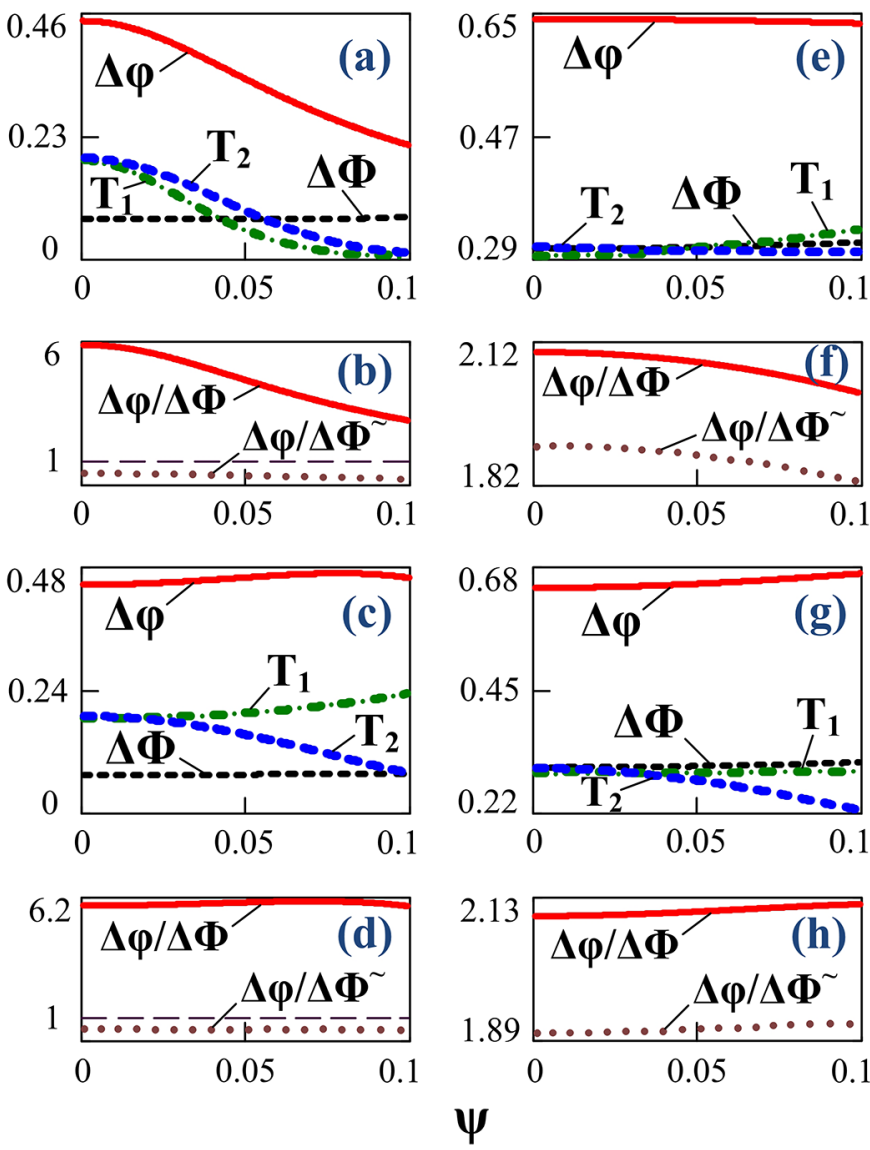

Fig. 8. Dependences of PSD $\Delta \varphi, \Delta \Phi$, relations $\Delta \varphi / \Delta \Phi, \Delta \varphi / \Delta \Phi^{\sim}$ and energy transmission coefficients $T_{1,2}$ of waves 1,2 on incidence angle $\psi$ for silver layers 1,3 , two different wavelengths and TM (a, b, e, f), TE (c, d, g, h) polarizations of the incident wave. The values of parameters are: $(\mathrm{a}-\mathrm{d}) \lambda=292 \mathrm{~nm}$, $d_{1,3}=125 \mathrm{~nm}, \quad d_{2}=19 \mathrm{~nm} ; \quad(\mathrm{e}-\mathrm{h}) \quad \lambda=650 \mathrm{~nm}, \quad d_{1,3}=9 \mathrm{~nm}$, $d_{2}=171 \mathrm{~nm}$. Figures b, d, f, h detail the data in figures a, c, e, $\mathrm{g}$, respectively. The black horizontal dashed line in figures $\mathrm{b}, \mathrm{d}$ characterizes equality to 1 of the corresponding quantities. Parameter $\psi$ is used as the abscissa axis for all the graphs. Values of $\psi$ (as $\left.\Delta \varphi, \Delta \Phi, \Delta \Phi^{\sim}\right)$ are given in the relative units of radians $/ \pi$.

TE polarizations of the exciting wave up to incidence angles of the order of $\psi=\pi / 20$ (the changes do not exceed several per cents, especially for TE waves and metallic layers 1,3$)$.

Electromagnetic field inside of the main layer for various parameters of the multilayer is characterized by the graphs in Figures 9 and 10. It is seen that the transition from "conventional" (Fig. 9a,c) to ENZ (Fig. 9b) or metallic (Fig. 9d) layers 1, 3 is accompanied by the significant amplification of the reflection (described by parameters $R_{21}, R_{23}$ ) of the waves on the boundaries $z=z_{1,2}$ inside of the layer for the definite thicknesses $d_{2}$. With that, the values of $d_{2}$ for the "peaks" of values of $R_{21}, R_{23}$ and $T_{1}$ for the corresponding graphs in Figure $9 \mathrm{~b}$ and Figure $2 \mathrm{f}$, Figure 9d and Figure 6d coincide. Near to the "peaks" of values of $R_{21}, R_{23}$ and $T_{1}$ (that is, when the optical interference effects are amplified in the main layer), the leap-frog change of the wave 1 phase takes place (Fig. 9b,d).
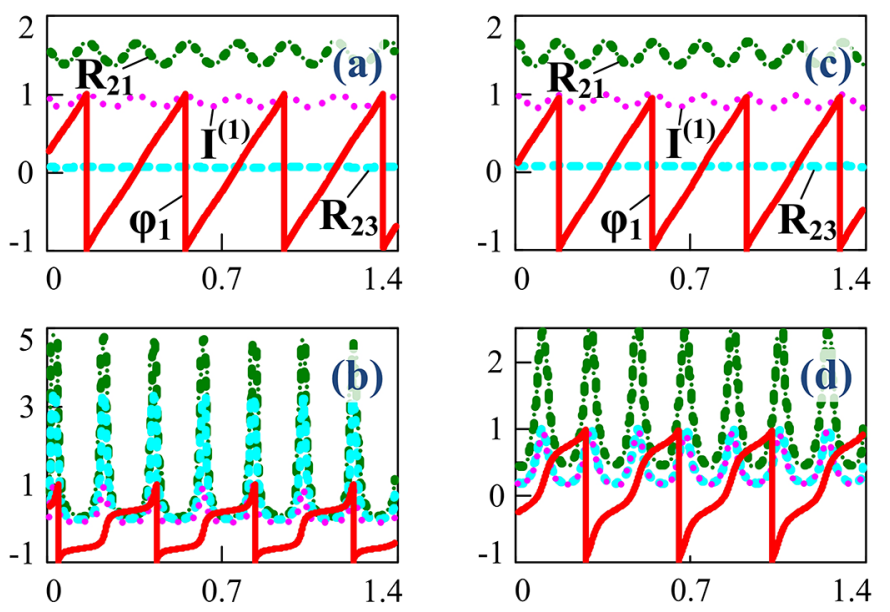

\section{$\mathbf{d}_{\mathbf{2}}, \boldsymbol{\mu m}$}

Fig. 9. Dependences of quantities $\varphi_{1}, R_{21}, R_{23}, I^{(1)}$ on the main layer thickness $d_{2}$ for various materials of layers 1,3 . The values of parameters are: $\varepsilon_{1}=\varepsilon_{3}=2+10^{-3} i \quad(\mathrm{a}, \mathrm{c}), 0.01+0.01 i \quad(\mathrm{~b})$, $-13+0.5 i(\mathrm{~d}) ; d_{1,3}=250 \mathrm{~nm}(\mathrm{a}, \mathrm{b}), 13 \mathrm{~nm}(\mathrm{c}, \mathrm{d}) ; \lambda=0.63 \mu \mathrm{m}$ $(\mathrm{a}, \mathrm{b}), 0.6 \mu \mathrm{m}(\mathrm{c}, \mathrm{d})$. Denotations of the curves in Figures b, $d$ are given in Figures a, c. $I^{(1)}=I^{T M(1)}\left(z=d_{1}+0.5 d_{2}\right)$ for all the graphs. Parameter $d_{2}$ is used as the abscissa axis for all the graphs.
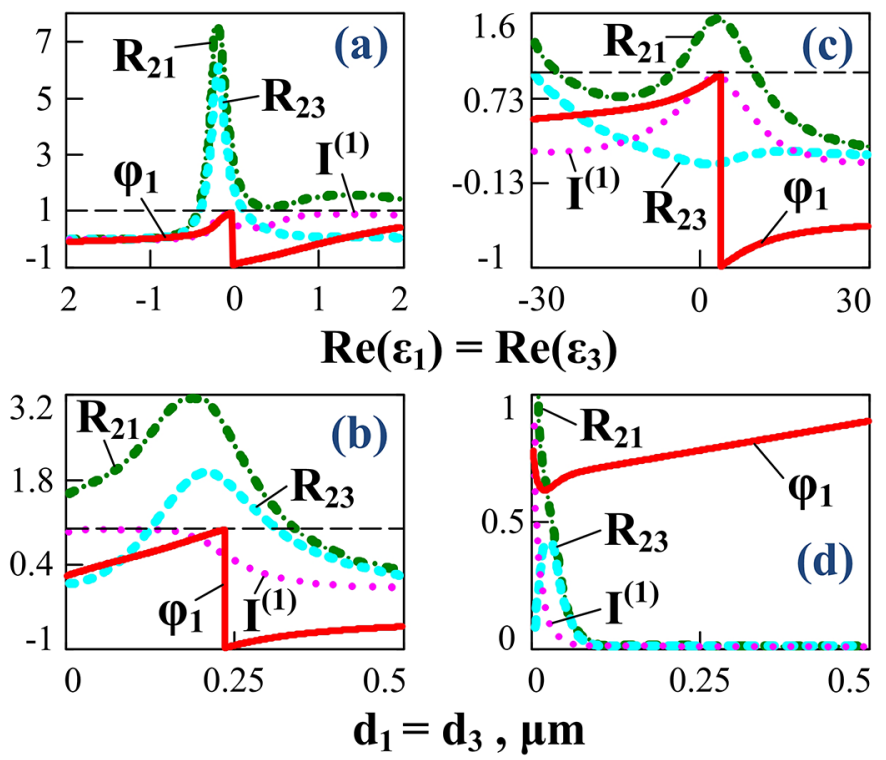

Fig. 10. Dependences of quantities $\varphi_{1}, R_{21}, R_{23}, I^{(1)}$ on parameters $\varepsilon_{1,3}^{\prime}(\mathrm{a}, \mathrm{c}), d_{1,3}(\mathrm{~b}, \mathrm{~d})$ for various materials of layers 1,3 . The values of parameters are: $\varepsilon_{1,3}^{\prime \prime}=0.01(\mathrm{a}, \mathrm{b}), 0.5(\mathrm{c}, \mathrm{d})$; $\varepsilon_{1,3}^{\prime}=0.01$ (b), $-13(\mathrm{~d}) ; d_{2}=39 \mathrm{~nm}(\mathrm{a}, \mathrm{b}), 156 \mathrm{~nm} \mathrm{(c,} \mathrm{d);}$ $d_{1,3}=250 \mathrm{~nm}(\mathrm{a}, \mathrm{b}), 13 \mathrm{~nm}(\mathrm{c}, \mathrm{d}) ; \lambda=0.63 \mu \mathrm{m}(\mathrm{a}, \mathrm{b}), 0.6 \mu \mathrm{m}$ $(\mathrm{c}, \mathrm{d}) . I^{(1)}=I^{T M(1)}\left(z=d_{1}+0.5 d_{2}\right)$ for all the graphs. The black horizontal dashed line in figs. a, b, c characterizes equality to 1 of the corresponding quantities.

As for Figure 2 data, the distances between the neighboring abrupt changes of $\varphi_{1}$ values from $\pi$ to $-\pi$ and maxima of functions $R_{21}\left(d_{2}\right), R_{23}\left(d_{2}\right)$ (Fig. 9b,d) are well described by values $\Delta d_{2}$ and $\Delta d_{2} / 2$, respectively. The values $R_{21}, R_{23}>1$ 
in Figures 9, 10 data characterize qualitatively reflection from the corresponding boundaries without taking into account the opposite waves interference inside of the main layer. For all the data in Figures 9 and 10, the normalized intensity of the resulting field inside of the main layer for Strip 1 (determining by Eq. (18) with account of the opposite waves interference) has the values $I^{T M(1)}<1$ (values of $I^{T M(1)}\left(z=d_{1}+0.5 d_{2}\right)$ are given in Figs. 9, 10). This condition points to the conservation of the energy fluxes balance in the main layer. So, Figure 9 data confirm the interference nature of the considered effects of PSD amplification for waves 1,2 at the multilayer (nanoresonator) output.

The graphs in Figure 10 illustrate the effect of parameters $\varepsilon_{1,3}^{\prime}, d_{1,3}$ on the energy fluxes and field intensity inside of the main layer. For ENZ layers 1, 3, the pronounced maxima of the functions characterizing dependences of quantities $R_{21}, R_{23}$ on parameters $\varepsilon_{1,3}^{\prime}$, $d_{1,3}$ (Fig. 10a,b) take place. For metallic layers 1, 3, such maxima are absent (the data in Fig. 10c for $\varepsilon_{1,3}^{\prime}<0$, Fig. 10d). When varying parameters $d_{1,3}$, the abrupt phase $\varphi_{1}$ changes take place for ENZ layers 1, 3 (Fig. 10b). For metallic layers 1,3 (Fig. 10d), the values of $R_{21}, R_{23}, I^{T M(1)}$ decrease quickly with growth of $d_{1,3}$. The graphs of quantity $I^{T M(1)}$ given in Figures 9,10 depend very weakly on the choice of coordinate $z$ of the point inside of the main layer. That is conditioned by the small value of parameter $\operatorname{Im}\left(\varepsilon_{2}^{(1)}\right)=10^{-3}$ determining small absorption of the radiation inside of the main layer. According to the numerical analysis results, dependences $I^{(1)}(z)$ enhance with the growth of absorption (for $\operatorname{Im}\left(\varepsilon_{2}^{(1)}\right)$ values of the order of $\left.10^{-2} \div 10^{-1}\right)$. With that, values of $I^{(1)}$ decrease with increasing $z$ from $d_{1}$ to $d_{1}+d_{2}$.

\subsection{Case of uniaxial main layer materials}

The data in Figure 11 correspond to ones in Figure 4c,e,f,h but for the case of an uniaxial material (with the different orientations of the optical axis for the strips, Fig. 1) of Strips 1, 2. It is seen that the main features of the considered effects also take place for the anisotropic components of the main layer. For ENZ layers 1, 3, there are pronounced maxima of the functions characterizing dependences of quantities $|\Delta \varphi|, \Delta \varphi / \Delta \Phi, \Delta \varphi / \Delta \Phi^{\sim}, T_{1,2}$ on parameters $d_{2}$ and $\varepsilon_{1,3}^{\prime}$ (Fig. 11). With that, conditions $\Delta \varphi / \Delta \Phi \gg 1, \Delta \varphi / \Delta \Phi^{\sim}>1$ take place (Fig. 11b, d). Small anisotropy values of the strips material $\left(\left|\varepsilon_{o}-\varepsilon_{e}\right|<0.5\right.$, as for the case of isotropic strips with small values $\left.\left|\varepsilon_{2}^{(1)}-\varepsilon_{2}^{(2)}\right|\right)$ lead to the growth of possible values $\Delta \varphi / \Delta \Phi, \Delta \varphi / \Delta \Phi^{\sim}$ (the data in Fig. 11b,d in comparison with ones in Fig. 4e,h). According to Figure 11 data, the parameters ranges are also possible for anisotropic materials of the strips when rather large transmission and amplification of waves 1, 2 PSD are realized simultaneously.

The data in Figure 12 illustrate impact of parameters $\varepsilon_{o, e}^{\prime}$ (the main layer material is chosen similarly to the data in Fig. 11) on the properties of the considered systems for the choice of ENZ (Fig. 12a,b) and metallic (Fig. 12c,d) layers 1, 3. The graphs in Figure 12a,b and c,d are obtained for the values of $d_{1,2,3}$ corresponding to the first maximum
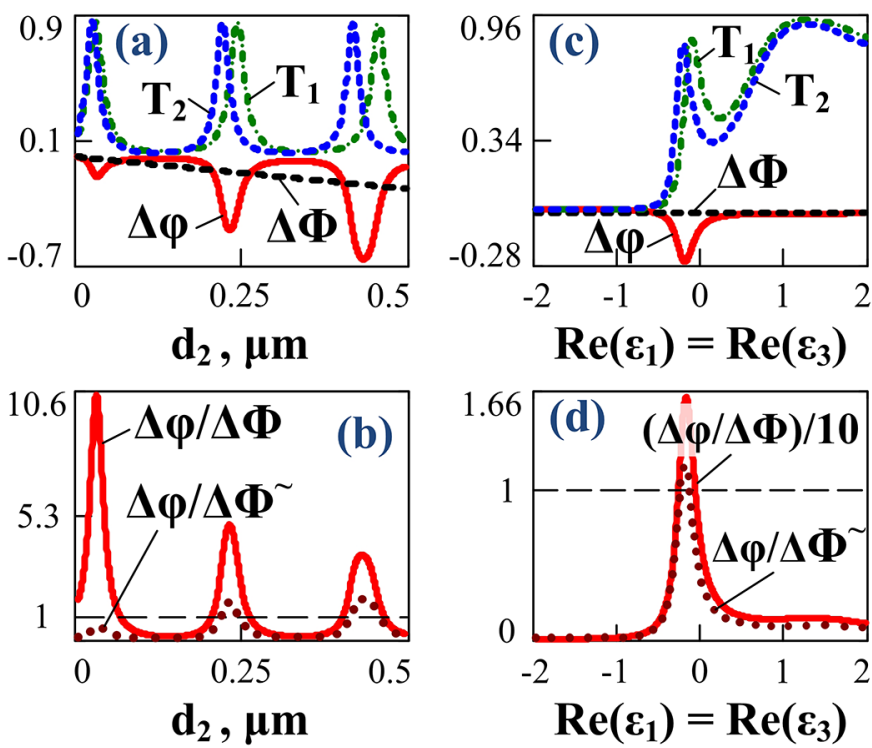

Fig. 11. Dependences of $\operatorname{PSD} \Delta \varphi, \Delta \Phi$, relations $\Delta \varphi / \Delta \Phi$, $\Delta \varphi / \Delta \Phi^{\sim}$ and energy transmission coefficients $T_{1,2}$ of waves 1,2 on the main layer thickness $d_{2}(\mathrm{a}, \mathrm{b})$, parameters $\varepsilon_{1,3}^{\prime}(\mathrm{c}, \mathrm{d})$ for a ENZ material of layers 1, 3 and anisotropic materials of the main layer. The values of parameters are: $\varepsilon_{1}=\varepsilon_{3}=0.01+0.01 i(\mathrm{a}, \mathrm{b})$; $\varepsilon_{1,3}^{\prime}=0.01(\mathrm{c}, \mathrm{d}) ; d_{2}=39 \mathrm{~nm}(\mathrm{c}, \mathrm{d})$. The values of parameters for all the graphs are: $\varepsilon_{o}^{(1,2)}=2.6+10^{-3} i ; \quad \varepsilon_{e}^{(1,2)}=2.2+10^{-3} i$; $d_{1,3}=250 \mathrm{~nm} ; \lambda=0.63 \mu \mathrm{m} ; \varepsilon_{\alpha, \beta}=1 ; \psi=0$. Figures $\mathrm{b}$ and d detail the data in Figures a and c, respectively. The black horizontal dashed line in Figures b, d characterizes equality to 1 of the corresponding quantities. TM polarization of the incident wave is used.

of functions $\Delta \varphi\left(d_{2}\right), \quad \Delta \varphi / \Delta \Phi\left(d_{2}\right), \quad \Delta \varphi / \Delta \Phi^{\sim}\left(d_{2}\right) \quad$ in Figures $11 \mathrm{a}, \mathrm{b}$ and $5 \mathrm{a}, \mathrm{b}$, respectively. It is seen that the graphs in Figure 12a and b, c and d are "symmetric" relatively to the vertical axis determined by the condition $\varepsilon_{o, e}^{\prime}=0$ (for the chosen TM polarization of the incident wave). Functions $\Delta \varphi\left(\varepsilon_{o, e}^{\prime}\right)$ increase (Fig. 12a,c) or decrease (Fig. 12b,d) monotonically for the investigated ranges of values $\varepsilon_{o, e}^{\prime}$. For all the cases considered in Figure 12, one of functions $T_{1,2}\left(\varepsilon_{o, e}^{\prime}\right)$ is constant and the other one is characterized by one (Fig. 12a,b) or two (Fig. 12c,d) maxima. The condition $\Delta \varphi / \Delta \Phi \gg 1$ can be satisfied for rather wide ranges of values $\varepsilon_{o, e}^{\prime}$ (with growth of $\left|\varepsilon_{o, e}^{\prime}\right|$ ) only for ENZ layers 1, 3 (Fig. 12a,b). For metallic layers 1, 3, this condition is also possible but for the narrow ranges of values $\varepsilon_{o, e}^{\prime}\left(\right.$ for $\varepsilon_{o, e}^{\prime}>0$ ) corresponding to the quick changes of functions $\Delta \varphi\left(\varepsilon_{o, e}^{\prime}\right)$ (Fig. 12c, d). With that, for "metallic" parameters of the main layer materials (for $\varepsilon_{o, e}^{\prime}<0$ ), the condition $\Delta \varphi \approx \Delta \Phi$ takes place (Fig. $12 \mathrm{c}, \mathrm{d}$ ).

\section{Conclusion}

The characteristic properties of transmission of electromagnetic waves through nanocomposite multilayers including ENZ MM or metallic spacer (upper and bottom) 

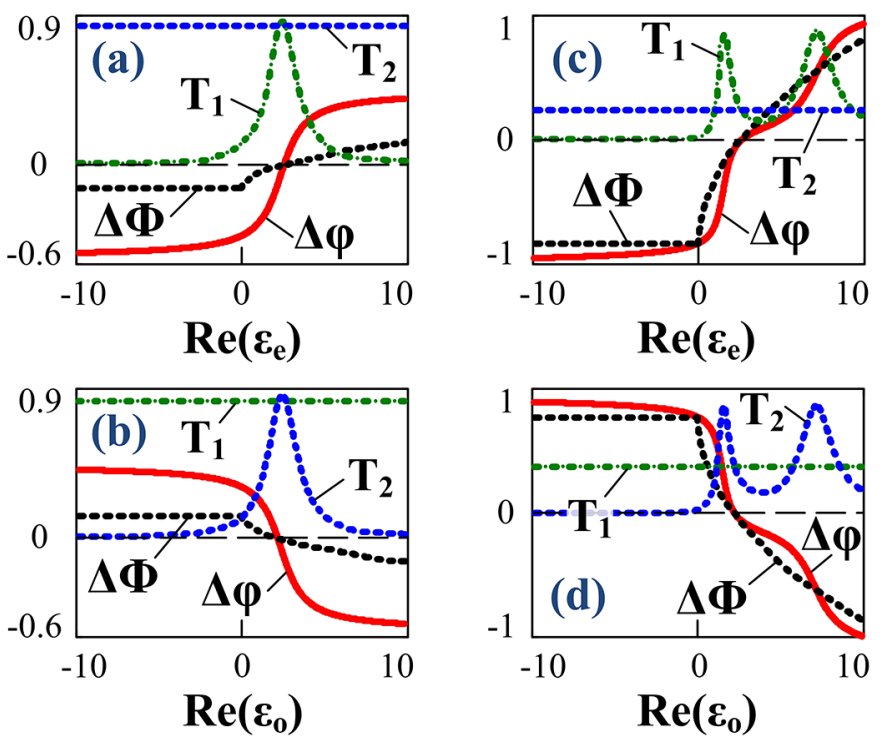

Fig. 12. Dependences of $\operatorname{PSD} \Delta \varphi, \Delta \Phi$, energy transmission coefficients $T_{1,2}$ of waves 1,2 on parameters $\operatorname{Re}\left(\varepsilon_{e}^{(1,2)}\right)$ (a, c) and $\operatorname{Re}\left(\varepsilon_{o}^{(1,2)}\right)(\mathrm{b}, \mathrm{d})$ for various materials of layers 1,3 and anisotropic materials of the main layer. The values of parameters are: (a) $\varepsilon_{1}=\varepsilon_{3}=0.01+0.01 i, \quad \varepsilon_{o}^{(1,2)}=2.6+10^{-3} i, \quad \operatorname{Im}\left(\varepsilon_{e}^{(1,2)}\right)=10^{-3}$, $d_{1,3}=250 \mathrm{~nm}, d_{2}=28 \mathrm{~nm}, \lambda=0.63 \mu \mathrm{m}$; (b) $\varepsilon_{1}=\varepsilon_{3}=0.01+0.01 i$, $\operatorname{Im}\left(\varepsilon_{o}^{(1,2)}\right)=10^{-3}, \varepsilon_{e}^{(1,2)}=2.2+10^{-3} i, d_{1,3}=250 \mathrm{~nm}, d_{2}=28 \mathrm{~nm}$, $\lambda=0.63 \mu \mathrm{m} ; \quad$ (c) $\quad \varepsilon_{1}=\varepsilon_{3}=-13+0.5 i, \quad \varepsilon_{o}^{(1,2)}=2.6+10^{-3} i$, $\operatorname{Im}\left(\varepsilon_{e}^{(1,2)}\right)=10^{-3}, d_{1,3}=13 \mathrm{~nm}, d_{2}=156 \mathrm{~nm}, \lambda=0.6 \mu \mathrm{m}$; (d) $\varepsilon_{1}=$ $\varepsilon_{3}=-13+0.5 i, \quad \operatorname{Im}\left(\varepsilon_{o}^{(1,2)}\right)=10^{-3}, \quad \varepsilon_{e}^{(1,2)}=2.2+10^{-3} i$, $d_{1,3}=13 \mathrm{~nm}, d_{2}=156 \mathrm{~nm}, \lambda=0.6 \mu \mathrm{m}$. The values of parameters for all the graphs are: $\varepsilon_{\alpha, \beta}=1 ; \psi=0$. The black horizontal dashed line in all the figures characterizes equality to 0 of the corresponding quantities. TM polarization of the incident wave is used.

layers and the main layer made of two alternating strips of low-absorbing dielectrics have been considered. The analytical modeling based on the exact solutions of electromagnetic boundary problems points to new possibilities of such nanoresonators usage as ultra-thin phase transformers of the transmitted radiation. Counterintuitively, but the "addition" to the system of two isotropic spacer layers (that are the same for rays 1, 2, Fig. 1, and do not change the "one pass" phase difference of the transmitted waves) with the definite properties gives the opportunities to increase significantly the exact PSD (by an order of magnitude or more) of the transmitted waves. The numerical and graphical analysis shows that the considered effects are most pronounced under subwavelength multilayer thicknesses and accompanied by the significant amplification of multibeam interference inside of the main layer.

Two main regimes of the composite nanoresonator usage for amplification of the transmitted waves PSD are revealed: (i) for ENZ spacers when the conditions $d_{2} \ll d_{1,3}$, $\Delta \Phi \ll \Delta \varphi \lesssim \Delta \Phi^{\sim}$ are realized; (ii) for metallic spacers when $d_{2} \gg d_{1,3}, \Delta \Phi, \Delta \Phi^{\sim}<\Delta \varphi$. These regimes were investigated for both model (ENZ and metallic) materials and silver (using two different wavelengths of the incident radiation) as spacer materials. For the considered ranges of the nanoresonator parameters, maximal values of PSD and transmission of the output waves are not realized simultaneously. But if the system is slightly tuned away from the maximal values, the parameter regions take place when PSD and transmission of the output waves are rather large for the whole multilayer thicknesses down to values of $\lambda / 2$ and less (e.g. this is confirmed by the Figs. 4, 5, 7 data). With that, the main layer thicknesses can be less than $\lambda / 15$ and $\lambda / 4$ for the regimes (i) and (ii), respectively. So, the conditions are possible when decreasing the whole system thickness down to significantly subwavelength values can lead to substantial increasing PSD of the transmitted waves. The investigated transmission features also take place when changing the system parameters such as possible presence of absorption (losses) of the nanoresonator components, anisotropy of the main layer materials, non-ideal realization of ENZ materials [41], oblique incidence of the exciting radiation (for small incidence angles).

Let us note that mechanisms of realization of large PSD for reflected and transmitted waves using the nanometric multilayers with ENZ components considered in [13,14] and the given paper are different. For reflection problems, the features of reflection of one of the waves from the ENZ substrate play a key role, and the interference effects are not main determining factors. For transmission, the effects of amplification of multibeam interference inside of the multilayer (that forms the nanoresonator) correspond to the conditions of obtaining large PSD of the transmitted waves. However, for both cases, the application of ENZ components for the considered layered systems leads to the quickly changing and extreme values of amplitude reflection or transmission coefficients depending on the system parameters. Namely that determines the main possibilities to amplify PSD of the output waves and decrease the whole system thickness.

The considered features of phase transformations of the transmitted waves can take place for various layered systems including the investigated nanoresonators or their modifications. The paper results also point to possibilities of exhibition of the investigated effects for the case when the main layer is originated by one optically anisotropic material. With that, the effective PSD amplification shall relate to the phase difference of proper waves (with different polarizations) in this material. One of the simplest methods to validate the obtained results is, apparently, a registration of the transmitted waves interference at the output of the considered systems, e.g. in the regimes of zero-order phase plates of subwavelength thicknesses.

The obtained results can be applied to develop various ultra-thin devices using phase transformation of transmitted waves, such as modulators, phase plates, filters, metasurfaces, nanoresonators, sensors, liquid crystals cells with reduced active material volumes, data processing system components, and for other nanophotonics applications. In general, together with the reflection problems analysis $[13,14]$, the modeling results point to the new and perspective approaches (that can be simply realized within 
the frames of existing thin films technologies) for realization of multilayered nanometric optical elements including ENZ components for effective phase transformation of both reflected and transmitted radiation. Moreover, the considered "amplification" of the transmitted waves phase differences using pair of nanometric ENZ or metallic layers could be of interest for phase-contrast microscopy methods [42-44]. One can expect that addition of such suitable layers on the substrate and cover glass (between which there is a transparent sample, a "phase object", e.g. biological cell) enhances weak phase contrasts of different regions of the sample and the image quality. That is of interest, in particular, for many biology and medicine applications of phase-contrast microscopy of transparent objects.

The author expresses the sincere appreciation to Dr Yuri Gritsai for the very fruitful discussions of the problem statement and results of this paper.

\section{Appendix}

The expressions for quantities $A_{ \pm}, B_{ \pm}^{(1)}, C_{ \pm}^{(1)}, D_{ \pm}, E_{ \pm}^{(1)}$, $F_{ \pm}^{(1)}$ in equations $(7)$ and (8) take the form

$$
\begin{gathered}
A_{ \pm}=(b-a) \exp \left(i k_{0} \eta_{1} d_{1}\right) \pm(b+a) \exp \left(-i k_{0} \eta_{1} d_{1}\right), \quad(\mathrm{A} 1) \\
B_{ \pm}^{(1)}=(1+c d) \exp \left(i k_{0} \eta_{3} d_{3}\right) \pm(1-c d) \exp \left(-i k_{0} \eta_{3} d_{3}\right),
\end{gathered}
$$

$$
C_{ \pm}^{(1)}=(1-c d) \exp \left(i k_{0} \eta_{3} d_{3}\right) \pm(1+c d) \exp \left(-i k_{0} \eta_{3} d_{3}\right)
$$

$D_{ \pm}=\left(\eta_{1}-\eta_{\alpha}\right) \exp \left(i k_{0} \eta_{1} d_{1}\right) \pm\left(\eta_{1}+\eta_{\alpha}\right) \exp \left(-i k_{0} \eta_{1} d_{1}\right)$

$$
\begin{aligned}
E_{ \pm}^{(1)}= & \left(\eta_{3}+\eta^{T E(1)}\right) \exp \left(i k_{0} \eta_{3} d_{3}\right) \\
& \pm\left(\eta_{3}-\eta^{T E(1)}\right) \exp \left(-i k_{0} \eta_{3} d_{3}\right), \\
F_{ \pm}^{(1)}= & \left(\eta_{3}-\eta^{T E(1)}\right) \exp \left(i k_{0} \eta_{3} d_{3}\right) \\
& \pm\left(\eta_{3}+\eta^{T E(1)}\right) \exp \left(-i k_{0} \eta_{3} d_{3}\right) .
\end{aligned}
$$

In a similar manner, we have for quantities $B_{ \pm}^{(2)}, C_{ \pm}^{(2)}$, $E_{ \pm}^{(2)}, F_{ \pm}^{(2)}$ in equations (9), (10)

$$
B_{ \pm}^{(2)}=(1+p d) \exp \left(i k_{0} \eta_{3} d_{3}\right) \pm(1-p d) \exp \left(-i k_{0} \eta_{3} d_{3}\right)
$$

$$
C_{ \pm}^{(2)}=(1-p d) \exp \left(i k_{0} \eta_{3} d_{3}\right) \pm(1+p d) \exp \left(-i k_{0} \eta_{3} d_{3}\right)
$$

$$
\begin{aligned}
E_{ \pm}^{(2)}= & \left(\eta_{3}+\eta^{T E(2)}\right) \exp \left(i k_{0} \eta_{3} d_{3}\right) \\
& \pm\left(\eta_{3}-\eta^{T E(2)}\right) \exp \left(-i k_{0} \eta_{3} d_{3}\right) \\
F_{ \pm}^{(2)}= & \left(\eta_{3}-\eta^{T E(2)}\right) \exp \left(i k_{0} \eta_{3} d_{3}\right) \\
& \pm\left(\eta_{3}+\eta^{T E(2)}\right) \exp \left(-i k_{0} \eta_{3} d_{3}\right)
\end{aligned}
$$

The expressions for quantities $A_{ \pm}, D_{ \pm}$(Eqs. (A1), (A4)) are also true for equations (9) and (10). The relations for the parameters in equations (A1)-(A10) are given in the main text.

\section{References}

1. A.M. Urbas et al., J. Optics 18, 093005 (2016)

2. N.M. Estakhri, A. Alù, JOSA B 33, A21 (2016)

3. T. Cui, S. Liu, L. Zhang, J. Mater. Chem. C 5, 3644 (2017)

4. I. Liberal, N. Engheta, Nat. Photonics 11, 149 (2017)

5. S.A. Tretyakov, J. Optics 19, 013002 (2017)

6. P. Genevet et al., Optica 4, 139 (2017)

7. L. Huang, S. Zhang, T. Zentgraf, Nanophotonics 7, 1169 (2018)

8. V-C. Su et al., Opt. Express 26, 13148 (2018)

9. V.S. Asadchy, A. Díaz-Rubio, S.A. Tretyakov, Nanophotonics 7, 1069 (2018)

10. M.A. Remnev, V.V. Klimov, Physics Uspekhi 61, 157 (2018)

11. M. Pu et al., Adv. Optical Mater. 7, 1801376 (2019)

12. S. Yoo, Q-H. Park, Nanophotonics 8, 249 (2019)

13. E.G. Starodubtsev, Mater. Res. Express 5, 126202 (2018)

14. E. Starodubtsev, EPJ Appl. Metamat. 6, 22 (2019)

15. R.W. Ziolkowski, E. Heyman, Phys. Rev. E 64, 056625 (2001)

16. A. Alù et al., Phys. Rev. B 75, 155410 (2007)

17. I.V. Lindell, A.H. Sihvola, PIER 89, 213 (2009)

18. E.G. Starodubtsev, Metamaterials 4, 32 (2010)

19. R. Maas et al., Nat. Photonics 7, 907 (2013)

20. N. Kinsey et al., Nat. Rev. Materials 1 (2019)

21. C. Valagiannopoulos et al., J. Appl. Phys. 121, 063102 (2017)

22. J. Kim et al., Nano Lett. 18, 740 (2018)

23. V. Caligiuri et al., Nanophotonics 8, 1505 (2019)

24. V. Caligiuri et al., Nano Lett. 19, 3151 (2019)

25. F.I. Fedorov, Optics of Anisotropic Media, 2nd edn. (Editorial URSS, Moscow, 2004 (in Russian))

26. M. Schadt, Annu. Rev. Mater. Sci. 27, 305 (1997)

27. D-K. Yang, S-T. Wu, Fundamentals of Liquid Crystal Devices, 2nd edn. (Wiley, 2014)

28. R. Häussler et al., Appl. Optics 56, F45 (2017)

29. Z. Yue et al., Nat. Commun. 8, 15354 (2017)

30. A.M. Shaltout et al., Nat. Commun. 9, 2673 (2018)

31. T.W. Ebbesen et al., Nature 391, 667 (1998)

32. E. Popov et al., Phys. Rev. B 62, 16100 (2000)

33. A. Degiron et al., Appl. Phys. Lett. 81, 4327 (2002)

34. D. Xiang et al., Opt. Commun. 284, 471 (2011)

35. A.S. Marathay, J.F. McCalmont, JOSA A 18, 2585 (2001)

36. V. Kivijärvi et al., New J. Phys. 17, 063019 (2015)

37. J.M. Coupland, N.I. Nikolaev, Opt. Express 27, 30380 (2019) 
38. C. Valagiannopoulos, PIER 71, 59 (2007)

39. A. Yariv, P. Yeh, Optical Electronics in Modern Communications, 6th edn. (Oxford University Press, 2007)

40. J. Smajic et al., J. Comput. Theor. Nanosci. 6, 1 (2009)
41. E. Starodubtsev, EPJ Appl. Metamat. 5, 1 (2018)

42. F. Zernike, Science, New Series 121, 345 (1955)

43. M. Teschke, S. Sinzinger, JOSA A 26, 1015 (2009)

44. K. Toda et al., Sci. Reports 9, 9957 (2019)

Cite this article as: Evgenii Starodubtsev, Features of transmission of electromagnetic waves through composite nanoresonators including epsilon-near-zero metamaterials, EPJ Appl. Metamat. 7, 1 (2020) 